\title{
A novel fractional structure of a multi-order quantum multi-integro-differential problem
}

\author{
Nguyen Duc Phuong ${ }^{1}$, Fethiye Muge Sakar ${ }^{2}$, Sina Etemad ${ }^{3}$ and Shahram Rezapour ${ }^{4,5,6 *}$ (D)
}

\author{
"Correspondence: \\ shahramrezapour@duytan.edu.vn; \\ sh.rezapour@mail.cmuh.org.tw; \\ sh.rezapour@azaruniv.ac.ir; \\ rezapourshahram@yahoo.ca \\ ${ }^{4}$ Institute of Research and \\ Development, Duy Tan University, \\ Da Nang 550000, Vietnam \\ ${ }^{5}$ Faculty of Natural Sciences, Duy \\ Tan University, Da Nang 550000, \\ Vietnam \\ Full list of author information is \\ available at the end of the article
}

\begin{abstract}
In the present research manuscript, we formulate a new generalized structure of the nonlinear Caputo fractional quantum multi-integro-differential equation in which such a multi-order structure of quantum integrals is considered for the first time. In fact, in the light of this type of boundary value problem equipped with the multi-integro-differential setting, one can simply study different cases of the existing usual integro-differential problems in the literature. In this direction, we utilize well-known analytical techniques to derive desired criteria which guarantee the existence of solutions for the proposed multi-order quantum multi-integro-differential problem. Further, some numerical examples are considered to examine our theoretical and analytical findings using the proposed methods.
\end{abstract}

MSC: Primary 34A08; secondary 34A12; 34A45

Keywords: Boundary value problem; Multi-integro-differential equation; Quantum calculus; The Caputo quantum derivative

\section{Introduction}

As years and even decades go by, the human beings need to be acquainted with different natural phenomena more and more. One possible way to achieve this purpose is to apply the logical techniques and tools available in mathematics, and particularly the mathematical operators, in the modeling of different processes. Various fractional operators have been formulated by different researchers, and their applicability is becoming increasingly apparent to researchers every day. In consequence, it is necessary that we derive and investigate various models of processes from all aspects by utilizing the fractional operators in boundary value problems. Some instances of the application of these operators can be found in applied sciences such as electrical circuits, medicine, biomathematics, etc. [1-6]. Moreover, the importance of this field implies that the researchers are interested in finding different aspects of the structure of the general fractional BVPs and some dynamical properties of their solutions. In this context, a lot of researchers have been studying many modern and general fractional models and relevant dynamical behaviors of this type of fractional BVPs (see, for example, [7-18]).

In 1910, Jackson [19] formulated a new field of the fractional calculus entitled the quantum fractional calculus or simply $q$-calculus. Shortly afterwards, Adams worked on the

(c) The Author(s) 2020. This article is licensed under a Creative Commons Attribution 4.0 International License, which permits use, sharing, adaptation, distribution and reproduction in any medium or format, as long as you give appropriate credit to the original author(s) and the source, provide a link to the Creative Commons licence, and indicate if changes were made. The images or other third party material in this article are included in the article's Creative Commons licence, unless indicated otherwise in a credit line to the material. If material is not included in the article's Creative Commons licence and your intended use is not permitted by statutory regulation or exceeds the permitted use, you will need to obtain permission directly from the copyright holder. To view a copy of this licence, visit http://creativecommons.org/licenses/by/4.0/. 
newly-defined quantum calculus and published some papers about $q$-difference equations [20-22]. At the same time, Carman and Starcher also continued this novel branch of the fractional calculus $[23,24]$. In the subsequent step, Trjitzinsky investigated analytic theory of linear quantum differential equations and also nonlinear quantum differential systems $[25,26]$. After the World War II, Abdi [27] studied certain quantum differential equations in 1962. Finally, Miller [28] combined quantum differential equations with Lie theory and investigated new theoretical results in this regard. By continuing this trend in the subsequent years, numerous researchers extended this field and obtained many interesting findings on the fractional quantum differential equations and inclusions (for more details, see [29-48]).

In 2013, Zhou and Liu [49], with the aid of Mönch's fixed point theorem along with an analytical technique based on the measure of weak noncompactness, turned to the following fractional quantum boundary problem:

$$
\left\{\begin{array}{l}
\mathcal{C}_{\mathfrak{D}_{q}^{\sigma}} \varpi(z)+\hat{h}_{*}(z, \varpi(z))=0, \quad z \in[0,1], \\
\varpi(0)=0, \quad \mathcal{C}_{\mathfrak{D}_{q}^{2} \varpi(0)=0,} \eta^{* \mathcal{C}} \mathfrak{D}_{q} \varpi(1)+\lambda^{*} \mathcal{C} \mathfrak{D}_{q}^{2} \varpi(1)=0,
\end{array}\right.
$$

such that $0<q<1,2<\sigma<3, \eta^{*}, \lambda^{*} \geq 0$, and $\hat{h}_{*}:[0,1] \times \mathbb{R} \rightarrow \mathbb{R}$ is supposed to be continuous.

In the usual fractional calculus setting, Niyom et al. [50] designed the following multiorder boundary problem in a new framework including Riemann-Liouville derivatives:

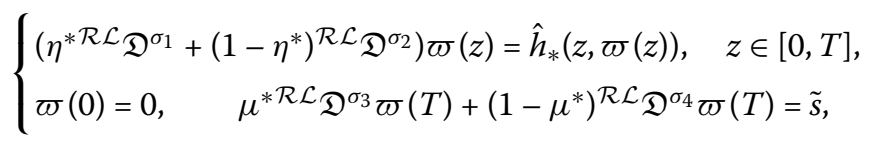

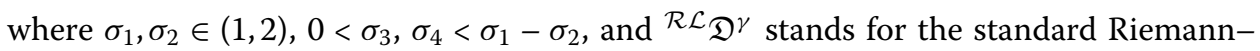
Liouville derivative of order $\gamma \in\left\{\sigma_{1}, \sigma_{2}, \sigma_{3}, \sigma_{4}\right\}$, and also $\eta^{*}, \mu^{*} \in(0,1], \tilde{s} \in \mathbb{R}$, and $\hat{h}_{*} \in$ $\mathcal{C}_{\mathbb{R}}([0, T] \times \mathbb{R})$ for $T>0$. Recently in 2019, Etemad, Ntouyas, and Ahmad [51] formulated a novel framework of the nonlinear fractional quantum integro-differential equation equipped with quantum integral conditions as follows;

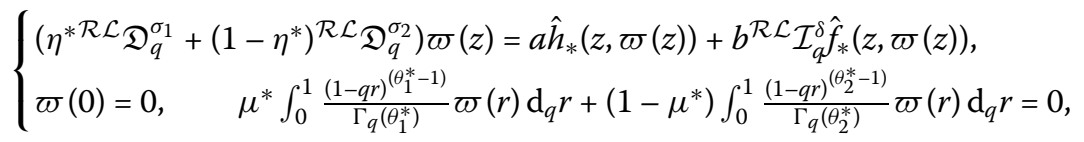

where $z \in[0,1], q \in(0,1), \sigma_{1}, \sigma_{2} \in(1,2)$ with $\sigma_{1}-\sigma_{2}>1, \eta^{*}, \mu^{*} \in(0,1), \theta_{1}^{*}, \theta_{2}^{*}>0, \delta \in(0,1)$, $a, b \in \mathbb{R}^{+}$, and ${ }^{\mathcal{R}} \mathcal{L}_{\mathfrak{D}_{q}^{\sigma}}$ stands for the Riemann-Liouville quantum derivative of order $\sigma$ while $\hat{h}_{*}, \hat{f}_{*}:[0,1] \times \mathbb{R} \rightarrow \mathbb{R}$ are supposed to be continuous functions.

Inspired by the aforementioned ideas given in the above-cited papers, we formulate a new generalized structure of the nonlinear Caputo fractional quantum multi-integrodifferential equation furnished with fractional multi-order quantum integrals conditions:

$$
\left\{\begin{array}{l}
\left(\eta^{* \mathcal{C}} \mathfrak{D}_{q}^{\sigma}-\left(\eta^{*}+1\right)^{\mathcal{R} \mathcal{L}} \mathcal{I}_{q}^{\delta_{1}^{*}}-\left(\eta^{*}+2\right)^{\mathcal{R} \mathcal{L}} \mathcal{I}_{q}^{\delta_{2}^{*}}\right) \varpi(z) \\
\quad=\lambda_{1}^{* \mathcal{R} \mathcal{L}} \mathcal{I}_{q}^{\gamma_{1}^{*}} \hat{h}_{*}(z, \varpi(z))+\lambda_{2}^{*} \mathcal{R} \mathcal{L} \mathcal{I}_{q}^{\gamma_{2}^{*}} \hat{f}_{*}(z, \varpi(z)), \\
\varpi(0)=0, \quad \mu^{* \mathcal{R} \mathcal{L}} \mathcal{I}_{q}^{\theta_{1}^{*}} \varpi(1)+\left(\mu^{*}+1\right)^{\mathcal{R} \mathcal{L}} \mathcal{I}_{q}^{\theta_{2}^{*}} \varpi(1)+\left(\mu^{*}+2\right)^{\mathcal{R} \mathcal{L}} \mathcal{I}_{q}^{\theta_{3}^{*}} \varpi(1)=0,
\end{array}\right.
$$


such that $z \in[0,1], \sigma \in(1,2), q \in(0,1), \delta_{1}^{*}, \delta_{2}^{*}, \gamma_{1}^{*}, \gamma_{2}^{*} \in(0,1), \theta_{1}^{*}, \theta_{2}^{*}, \theta_{3}^{*}>0$, and $\eta^{*}, \mu^{*}$ are nonzero real positive constants and $\lambda_{1}^{*}, \lambda_{2}^{*} \in \mathbb{R}^{\geq 0}$. Moreover, two operators, ${ }^{\mathcal{C}} \mathfrak{D}_{q}^{(\cdot)}$ and ${ }^{\mathcal{R}} \mathcal{I}_{q}^{(\cdot)}$, stand for the Caputo quantum derivative and the Riemann-Liouville quantum integral of given fractional orders, respectively. Also, both real-valued functions $\hat{h}_{*}, \hat{f}_{*}:[0,1] \times \mathbb{R} \rightarrow \mathbb{R}$ are supposed to be continuous. It is necessary that all researchers pay attention to that the proposed multi-order Caputo quantum multi-integro-differential equation has a novel and unique structure. In other words, the formulated structure for given fractional multi-integro-differential problem (1) includes one quantum derivative in the Caputo sense and also seven quantum integrals of the Riemann-Liouville type. This combined boundary problem covers many different special cases of various nonlinear integro-differential equations. Therefore, we emphasize that this kind of the Caputo quantum multi-integro-differential problem has not been investigated in the literature so far. In this direction, we apply well-known analytical techniques to derive desired criteria which guarantee the existence of solutions for the proposed Caputo quantum multiintegro-differential boundary problem (1).

The organization of the contents of the current manuscript is as follows. In the next section, some required notions in the context of the quantum calculus are assembled. Section 3 is devoted to establishing the main theorems in which the existence criteria can be obtained under some necessary conditions. In Sect. 4, numerical examples are considered to examine our theoretical and analytical findings by using the proposed methods.

\section{Preliminaries}

In this part of the present research manuscript, some required notions in the context of the quantum calculus are assembled. Let us assume that $q \in(0,1)$. For the given power function $\left(m_{1}-m_{2}\right)^{n}$ with $n \in \mathbb{N}_{0}$, its $q$-analogue is defined by $\left(m_{1}-m_{2}\right)^{(0)}=1$ and

$$
\left(m_{1}-m_{2}\right)^{(n)}=\prod_{k=0}^{n-1}\left(m_{1}-m_{2} q^{k}\right),
$$

such that $m_{1}, m_{2} \in \mathbb{R}$ and $\mathbb{N}_{0}:=\{0,1,2, \ldots\}$ [52]. Here, the constant $n=\sigma$ is supposed to be an arbitrary real number. In this case, one can define the $q$-analogue of mentioned power function $\left(m_{1}-m_{2}\right)^{n}$ in the $q$-fractional setting as follows:

$$
\left(m_{1}-m_{2}\right)^{(\sigma)}=m_{1}^{\sigma} \prod_{n=0}^{\infty} \frac{1-\left(\frac{m_{2}}{m_{1}}\right) q^{n}}{1-\left(\frac{m_{2}}{m_{1}}\right) q^{\sigma+n}}
$$

for $m_{1} \neq 0$. Notice that if we take $m_{2}=0$, then we reach an equality $m_{1}^{(\sigma)}=m_{1}^{\sigma}$ immediately [52]. For the given real number $m_{1} \in \mathbb{R}$, a $q$-number $\left[m_{1}\right]_{q}$ is considered as

$$
\left[m_{1}\right]_{q}=\frac{1-q^{m_{1}}}{1-q}=q^{m_{1}-1}+\cdots+q+1
$$

The quantum Gamma function, or simply the $q$-Gamma function, is provided by the following rule:

$$
\Gamma_{q}(z)=\frac{(1-q)^{(z-1)}}{(1-q)^{z-1}}
$$



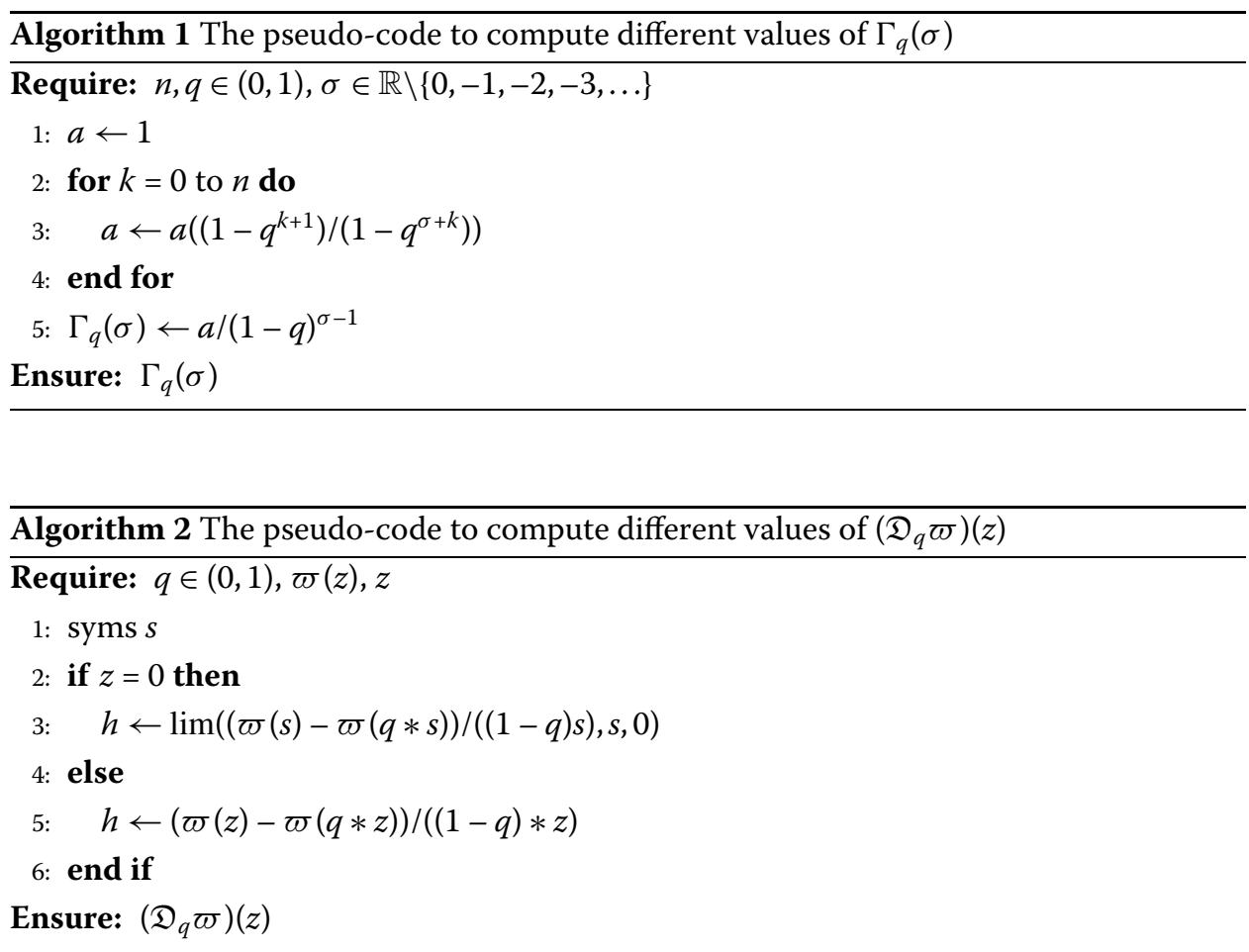

such that $z \in \mathbb{R} \backslash\{0,-1,-2, \ldots\}[19,52]$. It is notable that $\Gamma_{q}(z+1)=[z]_{q} \Gamma_{q}(z)$ is true [19]. In Algorithm 1, we provide a pseudo-code based on relations (2) and (3) to compute different values of the Gamma function in the quantum setting.

In the following, the quantum derivative of a real-valued continuous function $\varpi$ is defined by

$$
\left(\mathfrak{D}_{q} \varpi\right)(z)=\frac{\varpi(z)-\varpi(q z)}{(1-q) z}
$$

and also $\left(\mathfrak{D}_{q} \varpi\right)(0)=\lim _{z \rightarrow 0}\left(\mathfrak{D}_{q} \varpi\right)(z)$ [22]. One can simply extend the quantum derivative of a function $\varpi$ to arbitrary higher order by $\left(\mathfrak{D}_{q}^{n} \varpi\right)(z)=\mathfrak{D}_{q}\left(\mathfrak{D}_{q}^{n-1} \varpi\right)(z)$ for any $n \in \mathbb{N}$ [22]. It is obvious that $\left(\mathfrak{D}_{q}^{0} \varpi\right)(z)=\varpi(z)$. Similar to above, a pseudo-code based on (4) is provided to compute the quantum derivative of a function $\varpi$ in Algorithm 2.

The quantum integral of a real-valued continuous function $\varpi$ defined on $\left[0, m_{2}\right]$ is formulated by

$$
\left(\mathcal{I}_{q} \varpi\right)(z)=\int_{0}^{z} \varpi(r) \mathrm{d}_{q} r=z(1-q) \sum_{k=0}^{\infty} \varpi\left(z q^{k}\right) q^{k}, \quad z \in\left[0, m_{2}\right],
$$

provided that the series is absolutely convergent [22]. Similar to a quantum derivative, we can extend the quantum integral of a function $\varpi$ to arbitrary higher order by iterative rule $\left(\mathcal{I}_{q}^{n} \varpi\right)(z)=\mathcal{I}_{q}\left(\mathcal{I}_{q}^{n-1} \varpi\right)(z)$ for all $n \geq 1$ [22]. In addition, it is evident that $\left(\mathcal{I}_{q}^{0} \varpi\right)(z)=\varpi(z)$. Note that a pseudo-code based on (5) is provided to compute the quantum integral of a function $\varpi$ in Algorithm 3. At this moment, let us assume that $m_{1} \in\left[0, m_{2}\right]$. In this case, 


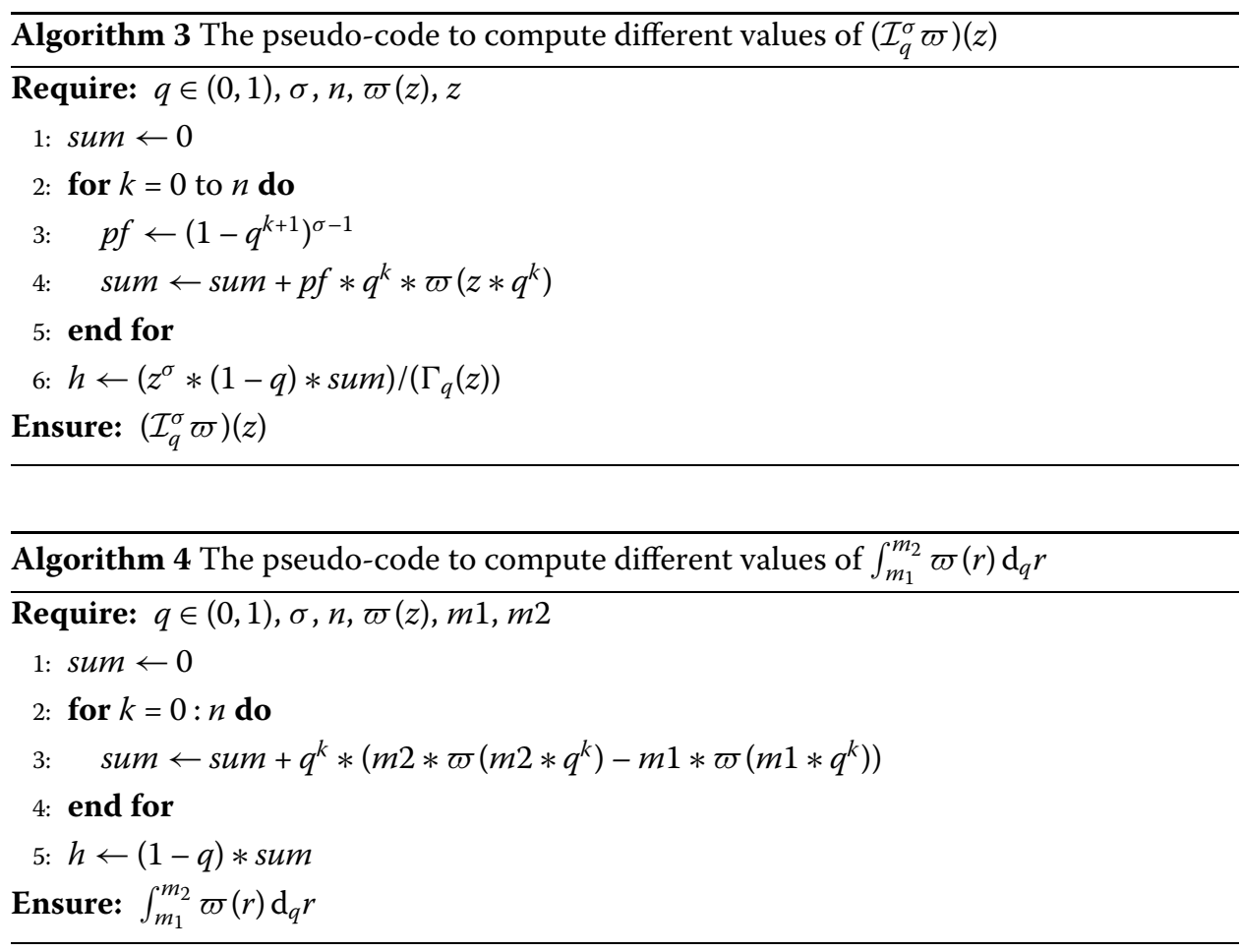

the quantum integral of the function $\varpi$ from $m_{1}$ to $m_{2}$ is defined as

$$
\begin{aligned}
\int_{m_{1}}^{m_{2}} \varpi(r) \mathrm{d}_{q} r & =\mathcal{I}_{q} \varpi\left(m_{2}\right)-\mathcal{I}_{q} \varpi\left(m_{1}\right) \\
& =\int_{0}^{m_{2}} \varpi(r) \mathrm{d}_{q} r-\int_{0}^{m_{1}} \varpi(r) \mathrm{d}_{q} r \\
& =(1-q) \sum_{k=0}^{\infty}\left[m_{2} \varpi\left(m_{2} q^{k}\right)-m_{1} \varpi\left(m_{1} q^{k}\right)\right] q^{k}
\end{aligned}
$$

if the right-hand side series has a finite value [22]. A pseudo-code based on (6) is provided to compute the quantum integral of a function $\varpi$ from $m_{1}$ to $m_{2}$ in Algorithm 4 .

Notice that if the function $\varpi$ is supposed to be continuous at the point $z=0$, then we have $\left(\mathcal{I}_{q} \mathfrak{D}_{q} \varpi\right)(z)=\varpi(z)-\varpi(0)$ [22]. Moreover, the equality $\left(\mathfrak{D}_{q} \mathcal{I}_{q} \varpi\right)(z)=\varpi(z)$ holds for each $z$. At this point, consider the real number $\sigma \geq 0$ so that $n-1<\sigma<n$, i.e., $n=$ $[\sigma]+1$. The Riemann-Liouville quantum integral for the given function $\varpi \in \mathcal{C}_{\mathbb{R}}([0,+\infty))$ is introduced by

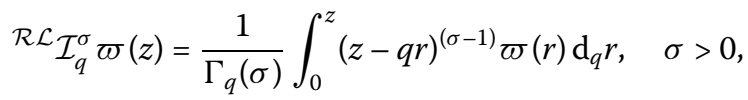

whenever the existing integral has finite value and ${ }^{\mathcal{R}} \mathcal{L}_{\mathcal{I}}^{0} \varpi(z)=\varpi(z)[53,54]$. Further, the semigroup property for this $q$-operator is valid, and so we have ${ }^{\mathcal{R} \mathcal{L}_{\mathcal{I}}} \mathcal{I}_{q}^{\sigma_{1}}\left({ }^{\mathcal{R}} \mathcal{I}_{q} \mathcal{I}_{q} \varpi\right)(z)=$ $\mathcal{R L}_{\mathcal{I}_{q}}^{\sigma_{1}+\sigma_{2}} \varpi(z)$ for $\sigma_{1}, \sigma_{2} \geq 0$ [55]. For $\theta \in(-1, \infty)$, the following property is valid:

$$
\mathcal{R L}_{\mathcal{I}_{q}^{\sigma}} z^{\theta}=\frac{\Gamma_{q}(\theta+1)}{\Gamma_{q}(\theta+\sigma+1)} z^{\theta+\sigma}, \quad z>0 .
$$



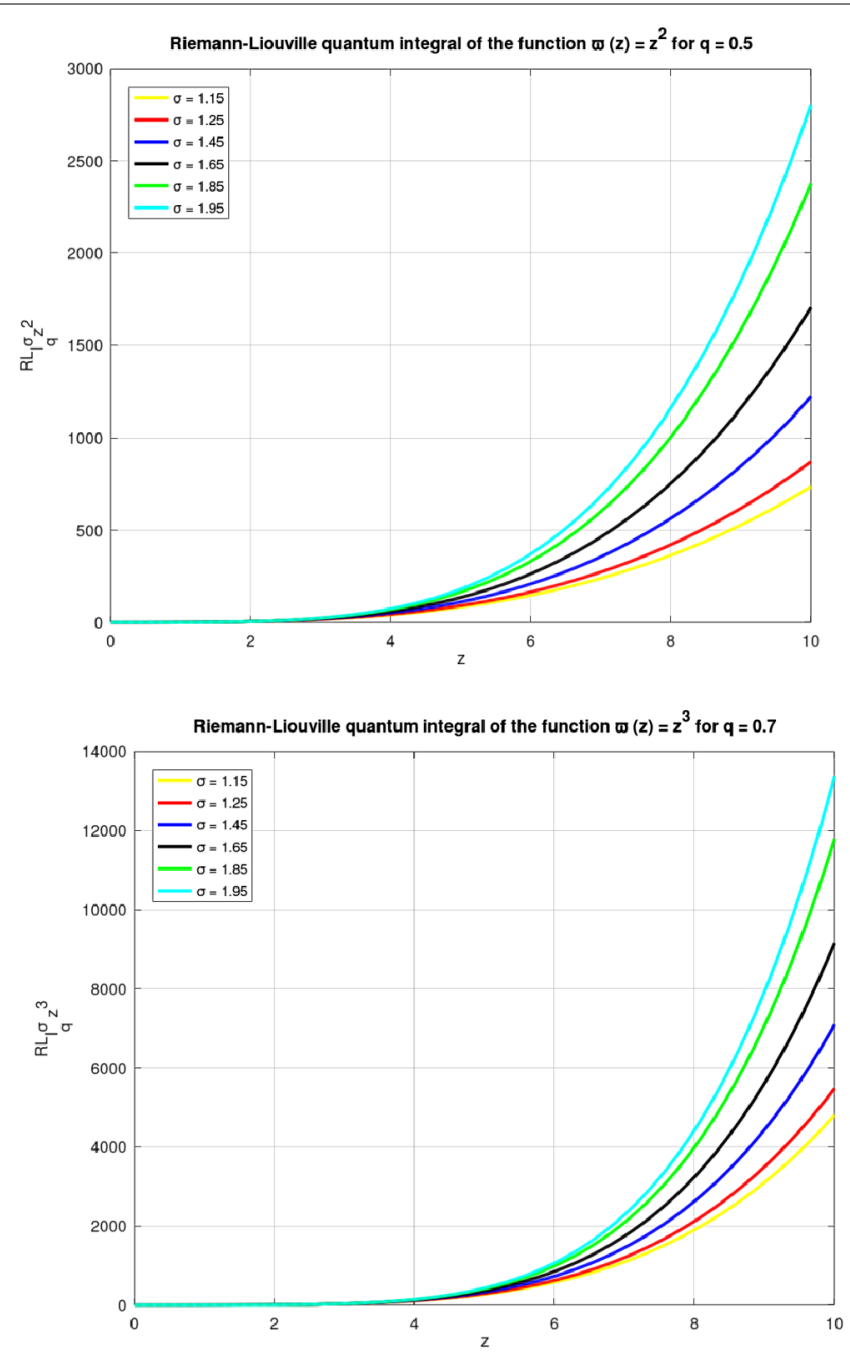

Figure 1 The Riemann-Liouville quantum integral of $\varpi(z)=z^{2}, z^{3}$ for $q=0.5,0.7$

It is evident that if we take $\theta=0$, then ${ }^{\mathcal{R}} \mathcal{L}_{\mathcal{I}_{q}^{\sigma}} 1(z)=\frac{1}{\Gamma_{q}(\sigma+1)} z^{\sigma}$ for any $z>0$. In the sequel, the Caputo quantum derivative for the given function $\varpi \in \mathcal{C}_{\mathbb{R}}^{(n)}([0,+\infty))$ is provided by

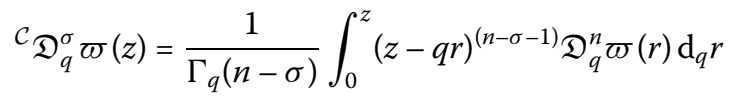

whenever the integral is finite-valued $[53,54]$. Notice that the following property is valid:

$$
\mathcal{C}_{\mathfrak{D}_{q}^{\sigma}} z^{\theta}=\frac{\Gamma_{q}(\theta+1)}{\Gamma_{q}(\theta-\sigma+1)} z^{\theta-\sigma}, \quad z>0 .
$$

It is evident that ${ }^{\mathcal{C}} \mathfrak{D}_{q}^{\sigma} 1(z)=0$ for any $z>0$. In Figs. 1 and 2 , the dynamical behavior of the Riemann-Liouville fractional quantum integral and the Caputo fractional quantum derivative can be observed on two given functions $\varpi(z)=z^{2}$ and $\varpi(z)=z^{3}$ for $q=0.5$ and $q=0.7$, respectively. 

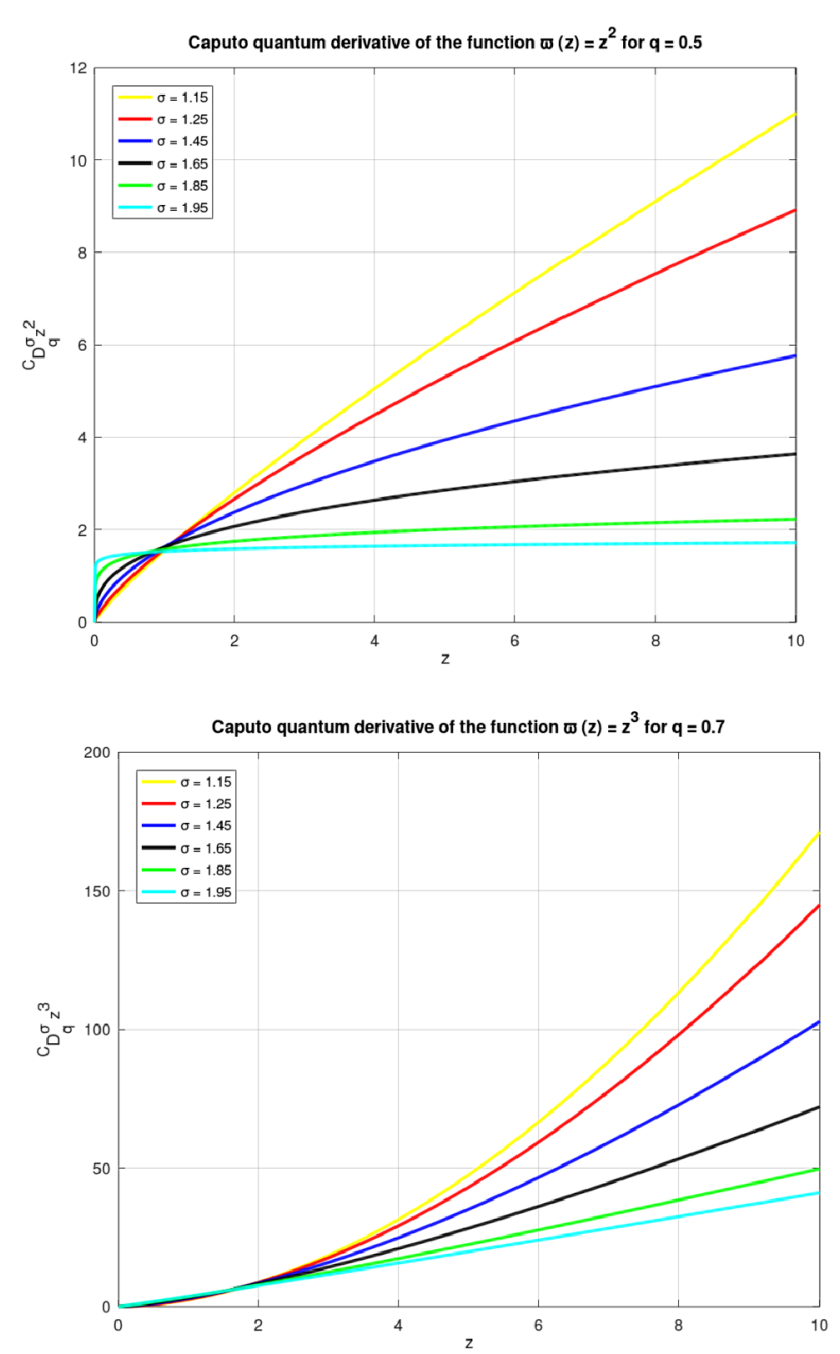

Figure 2 The Caputo quantum derivative of $\varpi(z)=z^{2}, z^{3}$ for $q=0.5,0.7$

Lemma 2.1 ([56]) Let $n-1<\sigma<n$. Then,

$$
\left({ }^{\mathcal{R}} \mathcal{L}_{q} \mathcal{I}_{q} \mathcal{C} \mathfrak{D}_{q}^{\sigma} \varpi\right)(z)=\varpi(z)-\sum_{k=0}^{n-1} \frac{z^{k}}{\Gamma_{q}(k+1)}\left(\mathfrak{D}_{q}^{k} \varpi\right)(0) .
$$

Due to the latter lemma, the general solution for the given fractional quantum differential equation ${ }^{\mathcal{C}} \mathfrak{D}_{q}^{\sigma} \varpi(z)=0$ is obtained by $\varpi(z)=\tilde{\alpha}_{0}+\tilde{\alpha}_{1} z+\tilde{\alpha}_{2} z^{2}+\cdots+\tilde{\alpha}_{n-1} z^{n-1}$ where $\tilde{\alpha}_{0}, \ldots, \tilde{\alpha}_{n-1}$ are arbitrary real numbers and $n=[\sigma]+1[56]$. Note that for every continuous function $\varpi$, by Lemma 2.1 , we have

$$
\left({ }^{\mathcal{R}} \mathcal{L}_{\mathcal{I}_{q}^{\sigma \mathcal{C}}} \mathfrak{D}_{q}^{\sigma} \varpi\right)(z)=\varpi(z)+\tilde{\alpha}_{0}+\tilde{\alpha}_{1} z+\tilde{\alpha}_{2} z^{2}+\cdots+\tilde{\alpha}_{n-1} z^{n-1}
$$

where $\tilde{\alpha}_{0}, \ldots, \tilde{\alpha}_{n-1}$ are constants which belong to $\mathbb{R}$ and $n=[\sigma]+1$ [56]. In what follows, some required fixed point theorems related to the proposed boundary problem are recalled. 
Theorem 2.2 (Krasnoselskii's fixed point theorem, [57]) Let $\mathbb{E}$ be a closed, convex, bounded, and nonempty subset of a Banach space $\mathfrak{W}$. Let $\mathbb{A}_{1}$ and $\mathbb{A}_{2}$ be two operators mapping $\mathbb{E}$ into $\mathfrak{W}$ so that the following statements are valid:

(i1) $\mathbb{A}_{1} \varpi_{1}+\mathbb{A}_{2} \varpi_{2} \in \mathbb{E}$, where $\varpi_{1}, \varpi_{2} \in \mathbb{E}$;

(i2) $\mathbb{A}_{1}$ is a contraction;

(i3) $\mathbb{A}_{2}$ is a continuous and compact operator.

Then there is an element $\varpi^{*} \in \mathbb{E}$ such that $\varpi^{*}=\mathbb{A}_{1} \varpi^{*}+\mathbb{A}_{2} \varpi^{*}$.

Theorem 2.3 (Nonlinear alternative for single-valued maps, [58]) Let $\mathfrak{W}$ be a Banach space, $\mathbb{M}$ a convex and closed subset of $\mathfrak{W}$, and $\mathbb{O}$ an open subset of $\mathbb{M}$ and $0 \in \mathbb{O}$. Moreover, let $\mathbb{A}: \overline{\mathbb{O}} \rightarrow \mathbb{M}$ be a continuous and compact operator (that is, $\mathbb{A}(\overline{\mathbb{O}})$ is a relatively compact subset of $\mathbb{M})$. Then either

(ii1) $\mathbb{A}$ has a fixed point in $\overline{\mathbb{O}}$; or

(ii2) there exists an element $\varpi^{*} \in \partial \mathbb{O}$ (as the boundary of the set $\mathbb{O}$ in $\mathbb{M}$ ) and $\hat{c} \in(0,1)$ with $\varpi^{*}=\hat{c} \mathbb{A}\left(\varpi^{*}\right)$.

Theorem 2.4 (Banach fixed point theorem, [59]) Let $\mathfrak{W}$ be a Banach space. Assume that $\mathbb{E} \subset \mathfrak{W}$ is closed and $\mathbb{A}: \mathbb{E} \rightarrow \mathbb{E}$ is a contraction. Then $\mathbb{A}$ is an operator having a fixed point in $\mathbb{E}$.

\section{Main results}

Let $\mathfrak{W}=\mathcal{C}_{\mathbb{R}}([0,1])$ be the space of all real-valued continuous functions on $[0,1]$. One can simply verify that the set $\mathfrak{W}$ will be a Banach space if we define the sup norm $\|\varpi\|_{\mathfrak{W}}=$ $\sup _{z \in[0,1]}|\varpi(z)|$ for all members $\varpi \in \mathfrak{W}$. At this point, we first provide the following structural lemma which characterizes the construction of solutions for the equivalent quantum integral equation related to the proposed quantum multi-integro-differential problem (1).

Lemma 3.1 Let $\Phi_{*} \in \mathfrak{W}, \sigma \in(1,2), \delta_{j}^{*} \in(0,1), \theta_{i}^{*}>0$ for $j=1,2$ and $i=1,2,3$. Also, let $\eta^{*}$, $\mu^{*}$ be nonzero real positive constants and consider the following nonzero positive constant:

$$
\tilde{\Xi}_{*}:=\frac{\mu^{*}}{\Gamma_{q}\left(\theta_{1}^{*}+2\right)}+\frac{\mu^{*}+1}{\Gamma_{q}\left(\theta_{2}^{*}+2\right)}+\frac{\mu^{*}+2}{\Gamma_{q}\left(\theta_{3}^{*}+2\right)} \neq 0
$$

Then the function $\varpi^{*}$ is a solution to the nonlinear Caputo quantum fractional problem

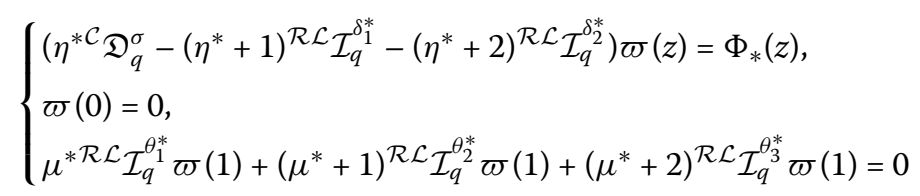

if and only if $\varpi^{*}$ is a solution to the fractional quantum integral equation

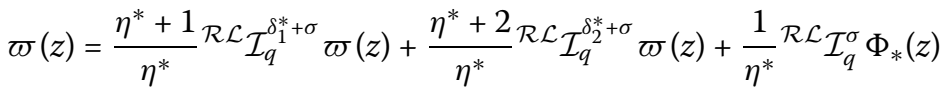

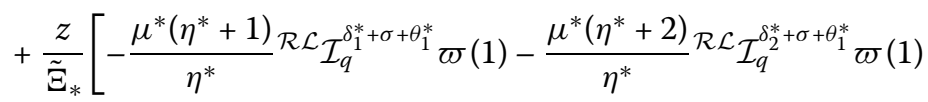

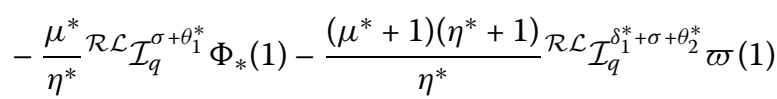




$$
\begin{aligned}
& -\frac{\left(\mu^{*}+1\right)\left(\eta^{*}+2\right)}{\eta^{*}} \mathcal{R} \mathcal{L}_{\mathcal{I}_{q}^{\delta}}^{\delta_{2}^{*}+\sigma+\theta_{2}^{*}} \varpi(1)
\end{aligned}
$$

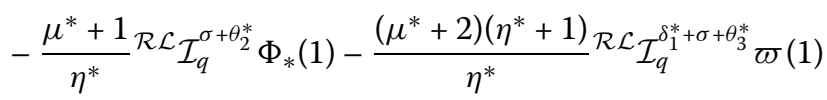

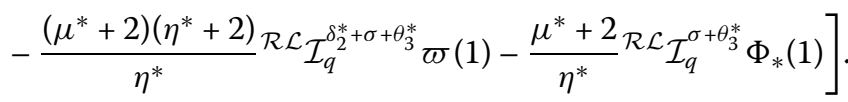

Proof At first, we regard the given function $\varpi^{*}$ as a solution for the Caputo quantum fractional problem (8). Then we get

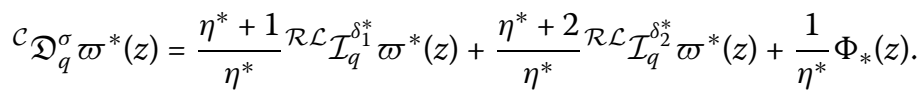

Taking fractional quantum integral in the Riemann-Liouville sense of order $\sigma$ on both sides of the latter equation, we reach

$$
\begin{aligned}
\varpi^{*}(z)= & \frac{\eta^{*}+1}{\eta^{*}} \mathcal{R} \mathcal{L}_{\mathcal{I}_{q}^{\delta_{1}^{*}+\sigma}} \varpi^{*}(z)+\frac{\eta^{*}+2}{\eta^{*}} \mathcal{R} \mathcal{L}_{\mathcal{I}_{q}^{\delta_{2}^{*}+\sigma}} \varpi^{*}(z)+\frac{1}{\eta^{*}} \mathcal{R} \mathcal{L}_{\mathcal{I}_{q}^{\sigma}} \Phi_{*}(z) \\
& +\tilde{\alpha}_{0}+\tilde{\alpha}_{1} z
\end{aligned}
$$

where $\tilde{\alpha}_{0}, \tilde{\alpha}_{1} \in \mathbb{R}$ are some constants that we need to find. It is immediately deduced that $\tilde{\alpha}_{0}=0$ by the first boundary condition and (10). On the other hand, by considering the properties of the Riemann-Liouville quantum integral, we have

$$
\begin{aligned}
\mathcal{R L}_{\mathcal{I}_{q}^{v}} \varpi^{*}(z)= & \frac{\eta^{*}+1}{\eta^{*}} \mathcal{R L}_{\mathcal{I}_{q}^{\delta_{1}^{*}+\sigma+v}} \varpi^{*}(z)+\frac{\eta^{*}+2}{\eta^{*}} \mathcal{R} \mathcal{L}_{\mathcal{I}_{q}^{\delta_{2}^{*}+\sigma+v}} \varpi^{*}(z) \\
& +\frac{1}{\eta^{*}} \mathcal{R L}_{\mathcal{I}_{q}^{\sigma+v}} \Phi_{*}(z)+\tilde{\alpha}_{0} \frac{z^{v}}{\Gamma_{q}(v+1)}+\tilde{\alpha}_{1} \frac{z^{v+1}}{\Gamma_{q}(v+2)}
\end{aligned}
$$

for $v \in\left\{\theta_{1}^{*}, \theta_{2}^{*}, \theta_{3}^{*}\right\}$. Then the second boundary condition (8) implies

$$
\begin{aligned}
& \tilde{\alpha}_{1}=\frac{1}{\tilde{\Xi}_{*}}\left[-\frac{\mu^{*}\left(\eta^{*}+1\right)}{\eta^{*}} \mathcal{R L}_{\mathcal{I}_{q}^{\delta_{1}^{*}+\sigma+\theta_{1}^{*}}} \varpi^{*}(1)\right.
\end{aligned}
$$

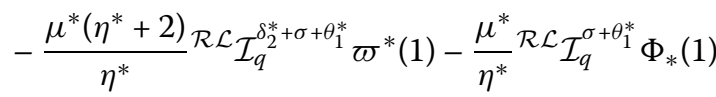

$$
\begin{aligned}
& -\frac{\left(\mu^{*}+1\right)\left(\eta^{*}+1\right)}{\eta^{*}} \mathcal{R} \mathcal{L}_{\mathcal{I}_{q}^{\delta}}^{\delta_{1}^{*}+\sigma+\theta_{2}^{*}} \varpi^{*}(1)-\frac{\left(\mu^{*}+1\right)\left(\eta^{*}+2\right)}{\eta^{*}} \mathcal{R} \mathcal{L}_{\mathcal{I}^{\delta}}^{\delta_{2}^{*}+\sigma+\theta_{2}^{*}} \varpi^{*}(1)
\end{aligned}
$$

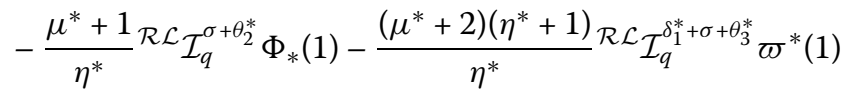

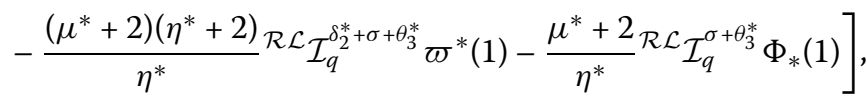

where $\tilde{\Xi}_{*} \neq 0$ is provided by (7). Eventually, we substitute both obtained values of $\tilde{\alpha}_{0}$ and $\tilde{\alpha}_{1}$ into (10). In this case, we observe that the function $\varpi^{*}$ satisfies the quantum integral equation (9), and so $\varpi^{*}$ is a solution for the mentioned integral equation. In the opposite direction, it is simple to confirm that $\varpi^{*}$ is a solution for the given nonlinear Caputo quantum fractional boundary problem (8) whenever $\varpi^{*}$ is regarded as a solution for the quantum integral equation (9). This completes the proof. 
Notation 3.2 From here onwards, for the sake of convenience in writing and computation, we consider the compact notations $\hat{h}_{*}(z, \varpi(z))=\hat{h}_{*}(z)$ and $\hat{f}_{*}(z, \varpi(z))=\hat{f}_{*}(z)$.

In the light of Lemma 3.1 and in relation to the proposed nonlinear Caputo fractional quantum multi-integro-differential equation (1), we construct an operator $\mathbb{A}: \mathfrak{W} \rightarrow \mathfrak{W}$ as follows:

$$
\begin{aligned}
& (\mathbb{A} \varpi)(z)=\frac{\eta^{*}+1}{\eta^{*}} \mathcal{R} \mathcal{L}_{\mathcal{I}_{q}^{1_{1}^{*}+\sigma}}^{{ }^{*}} \varpi(z)+\frac{\eta^{*}+2}{\eta^{*}} \mathcal{R} \mathcal{L}_{\mathcal{I}_{q}^{\delta_{2}^{*}+\sigma} \varpi(z)} \\
& +\frac{\lambda_{1}^{*}}{\eta^{*}} \mathcal{R} \mathcal{L}_{\mathcal{I}_{q}^{\gamma_{1}^{*}+\sigma}} \hat{h}_{*}(z)+\frac{\lambda_{2}^{*}}{\eta^{*}} \mathcal{R} \mathcal{L}_{\mathcal{I}_{q}^{\gamma_{2}^{*}+\sigma}} \hat{f}_{*}(z)
\end{aligned}
$$

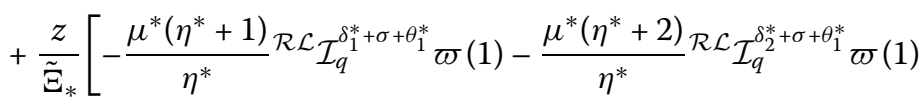

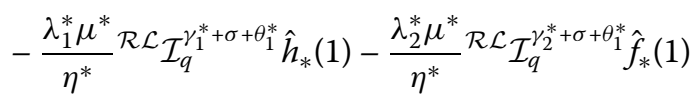

$$
\begin{aligned}
& -\frac{\left(\mu^{*}+1\right)\left(\eta^{*}+1\right)}{\eta^{*}} \mathcal{R} \mathcal{L}_{\mathcal{I}_{q}^{\delta}}^{\delta_{1}^{*}+\sigma+\theta_{2}^{*}} \varpi(1)
\end{aligned}
$$

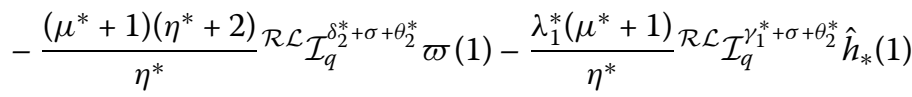

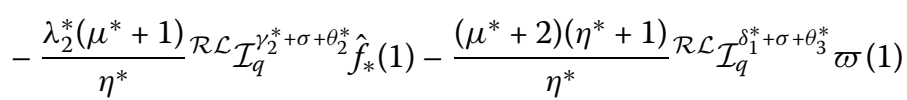

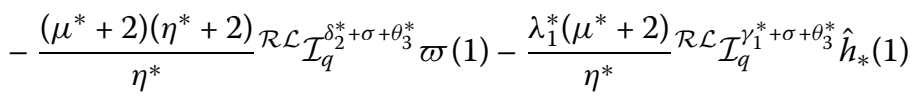

$$
\begin{aligned}
& \left.-\frac{\lambda_{2}^{*}\left(\mu^{*}+2\right)}{\eta^{*}} \mathcal{R} \mathcal{L}_{\mathcal{I}_{q}^{\gamma}}^{\gamma_{2}^{*}+\sigma+\theta_{3}^{*}} \hat{f}_{*}(1)\right]
\end{aligned}
$$

for each $\varpi \in \mathfrak{W}$ and $z \in[0,1]$. Consider the following constants which we will utilize these nonzero constants later:

$$
\begin{aligned}
\tilde{\Omega}_{*}^{(1)}:= & \frac{\eta^{*}+1}{\eta^{*} \Gamma_{q}\left(\delta_{1}^{*}+\sigma+1\right)}+\frac{\eta^{*}+2}{\eta^{*} \Gamma_{q}\left(\delta_{2}^{*}+\sigma+1\right)}+\frac{\mu^{*}\left(\eta^{*}+1\right)}{\eta^{*} \tilde{\Xi}_{*} \Gamma_{q}\left(\delta_{1}^{*}+\sigma+\theta_{1}^{*}+1\right)} \\
& +\frac{\mu^{*}\left(\eta^{*}+2\right)}{\eta^{*} \tilde{\Xi}_{*} \Gamma_{q}\left(\delta_{2}^{*}+\sigma+\theta_{1}^{*}+1\right)}+\frac{\left(\mu^{*}+1\right)\left(\eta^{*}+1\right)}{\eta^{*} \tilde{\Xi}_{*} \Gamma_{q}\left(\delta_{1}^{*}+\sigma+\theta_{2}^{*}+1\right)} \\
& +\frac{\left(\mu^{*}+1\right)\left(\eta^{*}+2\right)}{\eta^{*} \tilde{\Xi}_{*} \Gamma_{q}\left(\delta_{2}^{*}+\sigma+\theta_{2}^{*}+1\right)} \\
& +\frac{\left(\mu^{*}+2\right)\left(\eta^{*}+1\right)}{\eta^{*} \tilde{\Xi}_{*} \Gamma_{q}\left(\delta_{1}^{*}+\sigma+\theta_{3}^{*}+1\right)}+\frac{\left(\mu^{*}+2\right)\left(\eta^{*}+2\right)}{\eta^{*} \tilde{\Xi}_{*} \Gamma_{q}\left(\delta_{2}^{*}+\sigma+\theta_{3}^{*}+1\right)}, \\
\tilde{\Omega}_{*}^{(2)}:= & \frac{\lambda_{1}^{*}}{\eta^{*} \Gamma_{q}\left(\gamma_{1}^{*}+\sigma+1\right)}+\frac{\lambda_{1}^{*} \mu^{*}}{\eta^{*} \tilde{\Xi}_{*} \Gamma_{q}\left(\gamma_{1}^{*}+\sigma+\theta_{1}^{*}+1\right)} \\
& +\frac{\lambda_{1}^{*}\left(\mu^{*}+1\right)}{\eta^{*} \tilde{\Xi}_{*} \Gamma_{q}\left(\gamma_{1}^{*}+\sigma+\theta_{2}^{*}+1\right)}+\frac{\lambda_{1}^{*}\left(\mu^{*}+2\right)}{\eta^{*} \tilde{\Xi}_{*} \Gamma_{q}\left(\gamma_{1}^{*}+\sigma+\theta_{3}^{*}+1\right)}, \\
\tilde{\Omega}_{*}^{(3)}:= & \frac{\lambda_{2}^{*}}{\eta^{*} \Gamma_{q}\left(\gamma_{2}^{*}+\sigma+1\right)}+\frac{\lambda_{2}^{*} \mu^{*}}{\eta^{*} \tilde{\Xi}_{*} \Gamma_{q}\left(\gamma_{2}^{*}+\sigma+\theta_{1}^{*}+1\right)} \\
& +\frac{\lambda_{2}^{*}\left(\mu^{*}+1\right)}{\eta^{*} \tilde{\Xi}_{*} \Gamma_{q}\left(\gamma_{2}^{*}+\sigma+\theta_{2}^{*}+1\right)}+\frac{\lambda_{2}^{*}\left(\mu^{*}+2\right)}{\eta^{*} \tilde{\Xi}_{*} \Gamma_{q}\left(\gamma_{2}^{*}+\sigma+\theta_{3}^{*}+1\right)} .
\end{aligned}
$$


Now we are in the position to derive required existence criteria for the given nonlinear Caputo fractional quantum multi-integro-differential problem (1). To begin this process, we first invoke the well-known Krasnoselskii's fixed point theorem.

Theorem 3.3 Assume that two single-valued operators $\hat{h}_{*}:[0,1] \times \mathfrak{W} \rightarrow \mathfrak{W}$ and $\hat{f}_{*}$ : $[0,1] \times \mathfrak{W} \rightarrow \mathfrak{W}$ are continuous and also satisfy the following hypotheses:

(HK1) there exists a constant $\hat{b}^{*}>0$ such that for each $\varpi_{1}, \varpi_{2} \in \mathfrak{W}$ and for any $z \in[0,1]$, the inequality $\left|\hat{h}_{*}\left(z, \varpi_{1}\right)-\hat{h}_{*}\left(z, \varpi_{2}\right)\right| \leq \hat{b}^{*}\left|\varpi_{1}-\varpi_{2}\right|$ holds;

(HK2) there is a continuous function $\Upsilon$ on $[0,1]$ such that the inequality $\left|\hat{f}_{*}(z, \varpi)\right| \leq \Upsilon(z)$ is valid for any $z \in[0,1]$ and for every $\varpi \in \mathfrak{W}$.

Then the given nonlinear Caputo fractional quantum multi-integro-differential problem (1) has at least one solution on $[0,1]$ whenever $\tilde{\Omega}_{*}^{(1)}+\hat{b}^{*} \tilde{\Omega}_{*}^{(2)}<1$, where $\tilde{\Omega}_{*}^{(1)}$ and $\tilde{\Omega}_{*}^{(2)}$ are introduced by (12).

Proof We take $\|\Upsilon\|=\sup _{z \in[0,1]}|\Upsilon(z)|$ and construct $\mathbb{B}_{\tilde{r}}:=\{\varpi \in \mathfrak{W}:\|\varpi\| \leq \tilde{r}\}$ with

$$
\tilde{r} \geq \frac{\|\Upsilon\| \tilde{\Omega}_{*}^{(3)}+\hat{H}_{*} \tilde{\Omega}_{*}^{(2)}}{1-\left(\tilde{\Omega}_{*}^{(1)}+\hat{b}^{*} \tilde{\Omega}_{*}^{(2)}\right)}
$$

where $\hat{H}_{*}:=\sup _{z \in[0,1]}\left|\hat{h}_{*}(z, 0)\right|$ and $\tilde{\Omega}_{*}^{(1)}, \tilde{\Omega}_{*}^{(2)}$, and $\tilde{\Omega}_{*}^{(3)}$ are introduced by (12). As we know, the so-defined ball $\mathbb{B}_{\tilde{r}}$ is a bounded, convex, closed, and nonempty subset of the Banach space $\mathfrak{W}$. In addition, we consider an operator $\mathbb{A}: \mathfrak{W} \rightarrow \mathfrak{W}$ as in (11). In the light of Lemma 3.1, it is natural that the fixed point of $\mathbb{A}$ is considered as a solution for the nonlinear Caputo fractional quantum multi-integro-differential problem (1). To begin the proof, for any $z \in[0,1]$, we construct two operators $\mathbb{A}_{1}$ and $\mathbb{A}_{2}$ from $\mathbb{B}_{\tilde{r}}$ to $\mathfrak{W}$ as follows:

$$
\begin{aligned}
& \mathbb{A}_{1} \varpi(z)=\frac{\eta^{*}+1}{\eta^{*}} \mathcal{R} \mathcal{L}_{\mathcal{I}_{q}^{\delta^{*}+\sigma}} \varpi(z)+\frac{\eta^{*}+2}{\eta^{*}} \mathcal{R} \mathcal{L}_{\mathcal{I}_{q}^{\delta_{2}^{*}+\sigma}} \varpi(z)+\frac{\lambda_{1}^{*}}{\eta^{*}} \mathcal{L}_{\mathcal{I}_{q}^{\gamma_{1}^{*}+\sigma}} \hat{h}_{*}(z) \\
& +\frac{z}{\tilde{\Xi}_{*}}\left[-\frac{\mu^{*}\left(\eta^{*}+1\right)}{\eta^{*}} \mathcal{R} \mathcal{L}_{\mathcal{I}_{q}^{\delta}}^{\delta_{1}^{*}+\sigma+\theta_{1}^{*}} \varpi(1)-\frac{\mu^{*}\left(\eta^{*}+2\right)}{\eta^{*}} \mathcal{R} \mathcal{L}_{\mathcal{I}_{q}^{\delta *+\sigma+\theta_{1}^{*}}} \varpi(1)\right.
\end{aligned}
$$

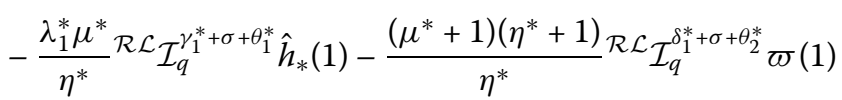

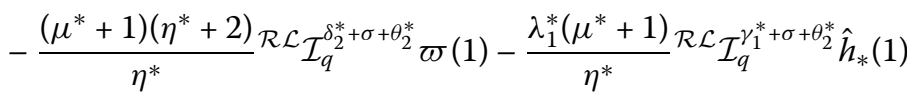

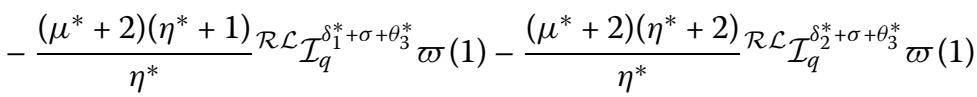

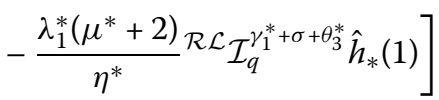

and

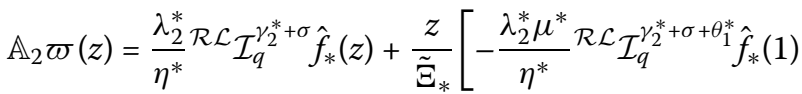

$$
\begin{aligned}
& \left.-\frac{\lambda_{2}^{*}\left(\mu^{*}+1\right)}{\eta^{*}} \mathcal{R} \mathcal{L}_{\mathcal{I}_{q}^{\gamma}}^{\gamma_{2}^{*}+\sigma+\theta_{2}^{*}} \hat{f}_{*}(1)-\frac{\lambda_{2}^{*}\left(\mu^{*}+2\right)}{\eta^{*}} \mathcal{R} \mathcal{L}_{\mathcal{I}^{\gamma}}^{\gamma_{2}^{*}+\sigma+\theta_{3}^{*}} \hat{f}_{*}(1)\right] .
\end{aligned}
$$


At first, due to hypothesis (HK1), we know that for any $z \in[0,1]$,

$$
\left|\hat{h}_{*}(z)\right|=\left|\hat{h}_{*}(z, \varpi(z))\right| \leq\left(\left|\hat{h}_{*}(z, \varpi(z))-\hat{h}_{*}(z, 0)\right|+\left|\hat{h}_{*}(z, 0)\right|\right) \leq \hat{b}^{*}|\varpi(z)|+\hat{H}_{*} .
$$

Also, hypothesis (HK2) implies that $\left|\hat{f}_{*}(z)\right|=\left|\hat{f}_{*}(z, \varpi)\right| \leq \Upsilon(z)$ for $z \in[0,1]$. Then for any elements $\varpi_{1}, \varpi_{2} \in \mathbb{B}_{\tilde{r}}$, one can write

$$
\begin{aligned}
& \left|\mathbb{A}_{1} \varpi_{1}(z)+\mathbb{A}_{2} \varpi_{2}(z)\right| \\
& \leq \frac{\eta^{*}+1}{\eta^{*}}{ }^{\mathcal{L}} \mathcal{L} \mathcal{I}_{q}^{\delta_{1}^{*+\sigma}}\left\|\varpi_{1}\right\|+\frac{\eta^{*}+2}{\eta^{*}}{ }^{\mathcal{R}} \mathcal{L}_{\mathcal{I}_{q}^{\delta_{2}^{*}+\sigma}}\left\|\varpi_{1}\right\|+\frac{\lambda_{1}^{*}}{\eta^{*}} \mathcal{R} \mathcal{L}_{\mathcal{I}_{q}^{\gamma_{1}^{*}+\sigma}}\left(\hat{b}^{*}\left\|\varpi_{1}\right\|+\hat{H}_{*}\right) \\
& +\frac{1}{\tilde{\Xi}_{*}}\left[\frac{\mu^{*}\left(\eta^{*}+1\right)}{\eta^{*}} \mathcal{R} \mathcal{L} \mathcal{I}_{q}^{\delta_{1}^{*}+\sigma+\theta_{1}^{*}}\left\|\varpi_{1}\right\|+\frac{\mu^{*}\left(\eta^{*}+2\right)}{\eta^{*}} \mathcal{R} \mathcal{L}_{\mathcal{I}_{q}^{\delta_{2}^{*}+\sigma+\theta_{1}^{*}}\left\|\varpi_{1}\right\|}\right. \\
& +\frac{\lambda_{1}^{*} \mu^{*}}{\eta^{*}} \mathcal{R} \mathcal{L}_{\mathcal{I}_{q}^{\gamma_{1}^{*}+\sigma+\theta_{1}^{*}}}\left(\hat{b}^{*}\left\|\varpi_{1}\right\|+\hat{H}_{*}\right) \\
& +\frac{\left(\mu^{*}+1\right)\left(\eta^{*}+1\right)}{\eta^{*}} \mathcal{R} \mathcal{L}_{\mathcal{I}_{q}^{\delta_{1}^{*}+\sigma+\theta_{2}^{*}}\left\|\varpi_{1}\right\|} \\
& +\frac{\left(\mu^{*}+1\right)\left(\eta^{*}+2\right)}{\eta^{*}} \mathcal{R} \mathcal{L}_{\mathcal{I}_{q}^{\delta^{*}+\sigma+\theta_{2}^{*}}\left\|\varpi_{1}\right\|} \\
& +\frac{\lambda_{1}^{*}\left(\mu^{*}+1\right)}{\eta^{*}} \mathcal{R} \mathcal{L}_{\mathcal{I}_{q}^{\gamma_{1}^{*}+\sigma+\theta_{2}^{*}}}\left(\hat{b}^{*}\left\|\varpi_{1}\right\|+\hat{H}_{*}\right)
\end{aligned}
$$

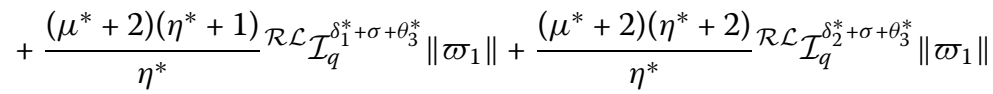

$$
\begin{aligned}
& \left.+\frac{\lambda_{1}^{*}\left(\mu^{*}+2\right)}{\eta^{*}} \mathcal{R} \mathcal{L} \mathcal{I}_{q}^{\gamma_{1}^{*}+\sigma+\theta_{3}^{*}}\left(\hat{b}^{*}\left\|\varpi_{1}\right\|+\hat{H}_{*}\right)\right] \\
& +\frac{\lambda_{2}^{*}}{\eta^{*}} \mathcal{R} \mathcal{L}_{\mathcal{I}_{q}^{\gamma_{2}^{*}+\sigma}}\|\Upsilon\|+\frac{1}{\tilde{\Xi}_{*}}\left[\frac{\lambda_{2}^{*} \mu^{*}}{\eta^{*}} \mathcal{R} \mathcal{L}_{\mathcal{I}_{q}^{\gamma_{2}^{*}+\sigma+\theta_{1}^{*}}\|\Upsilon\|}\right.
\end{aligned}
$$

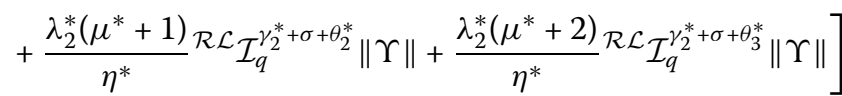

$$
\begin{aligned}
& =\left(\tilde{\Omega}_{*}^{(1)}+\hat{b}^{*} \tilde{\Omega}_{*}^{(2)}\right)\left\|\varpi_{1}\right\|+\tilde{\Omega}_{*}^{(3)}\|\Upsilon\|+\tilde{\Omega}_{*}^{(2)} \hat{H}_{*} \\
& \leq\left(\tilde{\Omega}_{*}^{(1)}+\hat{b}^{*} \tilde{\Omega}_{*}^{(2)}\right) \tilde{r}+\tilde{\Omega}_{*}^{(3)}\|\Upsilon\|+\tilde{\Omega}_{*}^{(2)} \hat{H}_{*} \leq \tilde{r} .
\end{aligned}
$$

The latter inequality demonstrates that $\left\|\mathbb{A}_{1} \varpi_{1}+\mathbb{A}_{2} \varpi_{2}\right\| \leq \tilde{r}$ and thus $\mathbb{A}_{1} \varpi_{1}+\mathbb{A}_{2} \varpi_{2} \in \mathbb{B}_{\tilde{r}}$ for each $\varpi_{1}, \varpi_{2} \in \mathbb{B}_{\tilde{r}}$. This also means that condition (i1) of Theorem 2.2 holds for both operators $\mathbb{A}_{1}$ and $\mathbb{A}_{2}$. At this point, we proceed to verify that $\mathbb{A}_{1}$ is a contraction. For arbitrary elements $\varpi_{1}, \varpi_{2} \in \mathbb{B}_{\tilde{r}}$ and $z \in[0,1]$, and in view of hypothesis (HK1), we have

$$
\begin{aligned}
& \left|\mathbb{A}_{1} \varpi_{1}(z)-\mathbb{A}_{1} \varpi_{2}(z)\right| \\
& \leq \frac{\eta^{*}+1}{\eta^{*}} \mathcal{L}_{\mathcal{I}_{q}^{\delta_{1}^{*}+\sigma}}\left|\varpi_{1}(z)-\varpi_{2}(z)\right| \\
& \quad+\frac{\eta^{*}+2}{\eta^{*}} \mathcal{R L}_{\mathcal{I}_{q}^{\delta_{2}^{*}+\sigma}}\left|\varpi_{1}(z)-\varpi_{2}(z)\right|+\frac{\lambda_{1}^{*}}{\eta^{*}} \mathcal{L}_{\mathcal{I}_{q}}^{\gamma_{1}^{*}+\sigma} \hat{b}^{*}\left|\varpi_{1}(z)-\varpi_{2}(z)\right| \\
& \quad+\frac{1}{\tilde{\Xi}_{*}}\left[\frac{\mu^{*}\left(\eta^{*}+1\right)}{\eta^{*}} \mathcal{R L}_{\mathcal{I}_{q}^{\delta_{1}^{*}+\sigma+\theta_{1}^{*}}}\left|\varpi_{1}(z)-\varpi_{2}(z)\right|+\frac{\mu^{*}\left(\eta^{*}+2\right)}{\eta^{*}} \mathcal{R L}_{\mathcal{I}_{q}^{\delta_{2}^{*}+\sigma+\theta_{1}^{*}}}\right.
\end{aligned}
$$




$$
\begin{aligned}
& \times\left|\varpi_{1}(z)-\varpi_{2}(z)\right|
\end{aligned}
$$

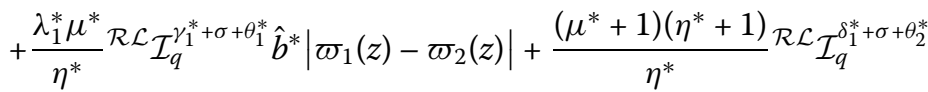

$$
\begin{aligned}
& \times\left|\varpi_{1}(z)-\varpi_{2}(z)\right| \\
& +\frac{\left(\mu^{*}+1\right)\left(\eta^{*}+2\right)}{\eta^{*}} \mathcal{R} \mathcal{L}_{\mathcal{I}_{q}^{\delta_{2}^{*}+\sigma+\theta_{2}^{*}}}\left|\varpi_{1}(z)-\varpi_{2}(z)\right| \\
& +\frac{\lambda_{1}^{*}\left(\mu^{*}+1\right)}{\eta^{*}} \mathcal{R L}_{\mathcal{I}_{q}^{\gamma^{*}+\sigma+\theta_{2}^{*}} \hat{b}^{*}}\left|\varpi_{1}(z)-\varpi_{2}(z)\right| \\
& +\frac{\left(\mu^{*}+2\right)\left(\eta^{*}+1\right)}{\eta^{*}} \mathcal{R L}_{\mathcal{I}_{q}{ }^{\delta^{*}+\sigma+\theta_{3}^{*}}}\left|\varpi_{1}(z)-\varpi_{2}(z)\right| \\
& +\frac{\left(\mu^{*}+2\right)\left(\eta^{*}+2\right)}{\eta^{*}} \mathcal{R} \mathcal{L}_{\mathcal{I}_{q}^{\delta_{2}^{*}+\sigma+\theta_{3}^{*}}}\left|\varpi_{1}(z)-\varpi_{2}(z)\right|
\end{aligned}
$$

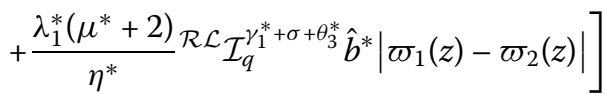

$$
\begin{aligned}
& =\left(\tilde{\Omega}_{*}^{(1)}+\hat{b}^{*} \tilde{\Omega}_{*}^{(2)}\right)\left\|\varpi_{1}-\varpi_{2}\right\| .
\end{aligned}
$$

In the light of the given hypothesis, we know that $\tilde{\Omega}_{*}^{(1)}+\hat{b}^{*} \tilde{\Omega}_{*}^{(2)}<1$. Thus we conclude that $\mathbb{A}_{1}$ is a contraction and so condition (i2) of Theorem 2.2 is valid for the operator $\mathbb{A}_{1}$.

In the sequel, we intend to verify the continuity of $\mathbb{A}_{2}$. To reach this goal, let us assume that $\left\{\varpi_{n}\right\}_{n \geq 1}$ is a convergent sequence belonging to the given ball $\mathbb{B}_{\tilde{r}}$ such that $\varpi_{n}$ approaches $\varpi$. Then for any $z \in[0,1]$, we obtain

$$
\begin{aligned}
\left|\mathbb{A}_{2} \varpi_{n}(z)-\mathbb{A}_{2} \varpi(z)\right| \leq & \frac{\lambda_{2}^{*}}{\eta^{*} \Gamma_{q}\left(\gamma_{2}^{*}+\sigma+1\right)}\left|\hat{f}_{*}\left(z, \varpi_{n}(z)\right)-\hat{f}_{*}(z, \varpi(z))\right| \\
& +\frac{\lambda_{2}^{*} \mu^{*}}{\eta^{*} \tilde{\Xi}_{*} \Gamma_{q}\left(\gamma_{2}^{*}+\sigma+\theta_{1}^{*}+1\right)}\left|\hat{f}_{*}\left(z, \varpi_{n}(z)\right)-\hat{f}_{*}(z, \varpi(z))\right| \\
& +\frac{\lambda_{2}^{*}\left(\mu^{*}+1\right)}{\eta^{*} \tilde{\Xi}_{*} \Gamma_{q}\left(\gamma_{2}^{*}+\sigma+\theta_{2}^{*}+1\right)}\left|\hat{f}_{*}\left(z, \varpi_{n}(z)\right)-\hat{f}_{*}(z, \varpi(z))\right| \\
& +\frac{\lambda_{2}^{*}\left(\mu^{*}+2\right)}{\eta^{*} \tilde{\Xi}_{*} \Gamma_{q}\left(\gamma_{2}^{*}+\sigma+\theta_{3}^{*}+1\right)}\left|\hat{f}_{*}\left(z, \varpi_{n}(z)\right)-\hat{f}_{*}(z, \varpi(z))\right| .
\end{aligned}
$$

But by the hypothesis, we know that the function $\hat{f}_{*}$ is continuous on $[0,1] \times \mathfrak{W}$, thus we find that $\left\|\mathbb{A}_{2} \varpi_{n}-\mathbb{A}_{2} \varpi\right\|$ approaches zero whenever $\varpi_{n} \rightarrow \varpi$. Therefore, we conclude that $\mathbb{A}_{2}$ is a continuous operator defined on $\mathbb{B}_{\tilde{r}}$. In the subsequent stage, we claim that the operator $\mathbb{A}_{2}$ is compact. To confirm this claim, we first check the uniform boundedness of $\mathbb{A}_{2}$. For given member $\varpi \in \mathbb{B}_{\tilde{r}}$ and $z \in[0,1]$, we may write

$$
\begin{aligned}
\left|\mathbb{A}_{2} \varpi(z)\right| \leq & \frac{\lambda_{2}^{*}}{\eta^{*}} \mathcal{R} \mathcal{L}_{\mathcal{I}_{q}^{\prime}}^{\gamma_{2}^{*}+\sigma}\left|\hat{f}_{*}(z)\right|+\frac{z}{\tilde{\Xi}_{*}}\left[\frac{\lambda_{2}^{*} \mu^{*}}{\eta^{*}} \mathcal{R} \mathcal{L}_{\mathcal{I}_{q}}^{\gamma_{2}^{*}+\sigma+\theta_{1}^{*}}\left|\hat{f}_{*}(1)\right|\right. \\
& \left.+\frac{\lambda_{2}^{*}\left(\mu^{*}+1\right)}{\eta^{*}} \mathcal{R} \mathcal{L}_{\mathcal{I}_{q}}^{\gamma_{2}^{*}+\sigma+\theta_{2}^{*}}\left|\hat{f}_{*}(1)\right|+\frac{\lambda_{2}^{*}\left(\mu^{*}+2\right)}{\eta^{*}} \mathcal{R} \mathcal{L}_{\mathcal{I}_{q}^{2}}^{\gamma_{2}^{*}+\sigma+\theta_{3}^{*}}\left|\hat{f}_{*}(1)\right|\right] \\
\leq & \|\Upsilon\|\left[\frac{\lambda_{2}^{*}}{\eta^{*} \Gamma_{q}\left(\gamma_{2}^{*}+\sigma+1\right)}+\frac{\lambda_{2}^{*} \mu^{*}}{\eta^{*} \tilde{\Xi}_{*} \Gamma_{q}\left(\gamma_{2}^{*}+\sigma+\theta_{1}^{*}+1\right)}\right.
\end{aligned}
$$




$$
\begin{aligned}
&+ \frac{\lambda_{2}^{*}\left(\mu^{*}+1\right)}{\eta^{*} \tilde{\Xi}_{*} \Gamma_{q}\left(\gamma_{2}^{*}+\sigma+\theta_{2}^{*}+1\right)} \\
&\left.+\frac{\lambda_{2}^{*}\left(\mu^{*}+2\right)}{\eta^{*} \tilde{\Xi}_{*} \Gamma_{q}\left(\gamma_{2}^{*}+\sigma+\theta_{3}^{*}+1\right)}\right] \\
&=\tilde{\Omega}_{*}^{(3)}\|\Upsilon\|,
\end{aligned}
$$

which illustrates that $\left\|\mathbb{A}_{2} \varpi\right\| \leq \tilde{\Omega}_{*}^{(3)}\|\Upsilon\|$ and $\mathbb{A}_{2}$ is uniformly bounded. Besides, we establish that $\mathbb{A}_{2}$ is an equicontinuous operator. To establish this result, we consider two elements $z, x \in[0,1]$ such that $z<x$. In fact, we shall verify that bounded sets are mapped to equicontinuous sets by the operator $\mathbb{A}_{2}$. Hence for every $\varpi \in \mathbb{B}_{\tilde{r}}$, we get

$$
\begin{aligned}
& \left|\mathbb{A}_{2} \varpi(x)-\mathbb{A}_{2} \varpi(z)\right| \\
& \leq \frac{\lambda_{2}^{*}}{\eta^{*} \Gamma_{q}\left(\gamma_{2}^{*}+\sigma\right)} \int_{0}^{z}\left[(x-q r)^{\left(\gamma_{2}^{*}+\sigma-1\right)}-(z-q r)^{\left(\gamma_{2}^{*}+\sigma-1\right)}\right]\left|\hat{f}_{*}(r, \varpi(r))\right| \mathrm{d}_{q} r \\
& +\frac{\lambda_{2}^{*}}{\eta^{*} \Gamma_{q}\left(\gamma_{2}^{*}+\sigma\right)} \int_{z}^{x}(x-q r)^{\left(\gamma_{2}^{*}+\sigma-1\right)}\left|\hat{f}_{*}(r, \varpi(r))\right| \mathrm{d}_{q} r \\
& +\frac{(x-z)}{\tilde{\Xi}_{*}}\left[\frac{\lambda_{2}^{*} \mu^{*}}{\eta^{*} \Gamma_{q}\left(\gamma_{2}^{*}+\sigma+\theta_{1}^{*}\right)} \int_{0}^{1}(1-q r)^{\left(\gamma_{2}^{*}+\sigma+\theta_{1}^{*}-1\right)}\left|\hat{f}_{*}(r, \varpi(r))\right| \mathrm{d}_{q} r\right. \\
& +\frac{\lambda_{2}^{*}\left(\mu^{*}+1\right)}{\eta^{*} \Gamma_{q}\left(\gamma_{2}^{*}+\sigma+\theta_{2}^{*}\right)} \int_{0}^{1}(1-q r)^{\left(\gamma_{2}^{*}+\sigma+\theta_{2}^{*}-1\right)}\left|\hat{f}_{*}(r, \varpi(r))\right| \mathrm{d}_{q} r \\
& \left.+\frac{\lambda_{2}^{*}\left(\mu^{*}+2\right)}{\eta^{*} \Gamma_{q}\left(\gamma_{2}^{*}+\sigma+\theta_{3}^{*}\right)} \int_{0}^{1}(1-q r)^{\left(\gamma_{2}^{*}+\sigma+\theta_{3}^{*}-1\right)}\left|\hat{f}_{*}(r, \varpi(r))\right| \mathrm{d}_{q} r\right] .
\end{aligned}
$$

We find that the right-hand side of the obtained inequality is not dependent on $\varpi \in \mathbb{B}_{\tilde{r}}$ and also approaches 0 when $z$ tends to $x$. In consequence, we realize that $\mathbb{A}_{2}$ is equicontinuous. Hence, it is concluded that $\mathbb{A}_{2}$ is a relatively compact operator on $\varpi \in \mathbb{B}_{\tilde{r}}$ and thus the Arzelá-Ascoli theorem implies that $\mathbb{A}_{2}$ is completely continuous, and eventually $\mathbb{A}_{2}$ is a compact operator on the given ball $\varpi \in \mathbb{B}_{\tilde{r}}$. Therefore condition (i3) of Theorem 2.2 is valid for the operator $\mathbb{A}_{2}$. In consequence, all three hypotheses of Theorem 2.2 are valid for both single-valued operators $\mathbb{A}_{1}$ and $\mathbb{A}_{2}$. Therefore Theorem 2.2 implies that the given nonlinear Caputo fractional quantum multi-integro-differential problem (1) has at least one solution on the interval $[0,1]$, and so this completes the proof.

Leray-Schauder nonlinear alternative theorem is another analytical tool by which we will be able to derive our desired existence criteria for the mentioned nonlinear Caputo fractional quantum multi-integro-differential problem (1).

Theorem 3.4 Let the functions $\hat{h}_{*}:[0,1] \times \mathfrak{W} \rightarrow \mathfrak{W}$ and $\hat{f}_{*}:[0,1] \times \mathfrak{W} \rightarrow \mathfrak{W}$ be continuous and satisfy the following assumptions:

(HK3) there exist two functions $\Phi_{1}, \Phi_{2} \in \mathcal{C}_{\mathbb{R}^{+}}([0,1])$ along with two continuous nondecreasing functions $\Psi_{1}, \Psi_{2}:[0, \infty) \rightarrow(0, \infty)$ such that for any $(z, \varpi) \in[0,1] \times \mathfrak{W}$, we have

$$
\left|\hat{h}_{*}(z, \varpi)\right| \leq \Phi_{1}(z) \Psi_{1}(|\varpi|) \quad \text { and } \quad\left|\hat{f}_{*}(z, \varpi)\right| \leq \Phi_{2}(z) \Psi_{2}(|\varpi|) ;
$$


(HK4) there exists a real constant $\mathfrak{N}>0$ such that $\tilde{\Omega}_{*}^{(1)}<1$ and

$$
\frac{\left(1-\tilde{\Omega}_{*}^{(1)}\right) \mathfrak{N}}{\tilde{\Omega}_{*}^{(2)}\left\|\Phi_{1}\right\| \Psi_{1}(\mathfrak{N})+\tilde{\Omega}_{*}^{(3)}\left\|\Phi_{2}\right\| \Psi_{2}(\mathfrak{N})}>1
$$

where $\tilde{\Omega}_{*}^{(1)}, \tilde{\Omega}_{*}^{(2)}$, and $\tilde{\Omega}_{*}^{(3)}$ are given by (12).

Then the nonlinear Caputo fractional quantum multi-integro-differential problem (1) has at least one solution on $[0,1]$.

Proof To reach the desired conclusion, we check all the hypotheses of Leray-Schauder nonlinear alternative (Theorem 2.3) in the subsequent steps. At first, we are going to show that the operator $\mathbb{A}$ defined by (11) maps bounded sets (i.e., balls) into bounded sets in $\mathfrak{W}$. For a positive real number $\tilde{R}$, construct a bounded ball $\mathbb{B}_{\tilde{R}}=\{\varpi \in \mathfrak{W}:\|\varpi\| \leq \tilde{R}\}$ in $\mathfrak{W}$. Then for any $z \in[0,1]$ and in view of hypothesis (HK3), we can write

$$
\begin{aligned}
& |\mathbb{A} \varpi(z)|
\end{aligned}
$$

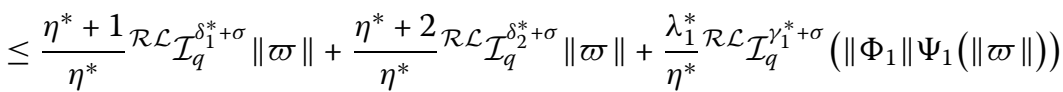

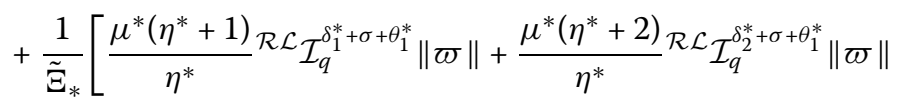

$$
\begin{aligned}
& +\frac{\lambda_{1}^{*} \mu^{*}}{\eta^{*}} \mathcal{R} \mathcal{L}_{\mathcal{I}_{q}^{\gamma_{1}^{*}+\sigma+\theta_{1}^{*}}}\left(\left\|\Phi_{1}\right\| \Psi_{1}(\|\varpi\|)\right)+\frac{\left(\mu^{*}+1\right)\left(\eta^{*}+1\right)}{\eta^{*}} \mathcal{R} \mathcal{L}_{\mathcal{I}_{q}^{\delta_{1}^{*}+\sigma+\theta_{2}^{*}}\|\varpi\|} \\
& +\frac{\left(\mu^{*}+1\right)\left(\eta^{*}+2\right)}{\eta^{*}} \mathcal{R} \mathcal{L}_{\mathcal{I}_{q}^{\delta_{2}^{*}+\sigma+\theta_{2}^{*}}\|\varpi\|} \\
& +\frac{\lambda_{1}^{*}\left(\mu^{*}+1\right)}{\eta^{*}} \mathcal{R} \mathcal{L}_{\mathcal{I}_{q}^{\gamma}}^{\gamma_{1}^{*}+\sigma+\theta_{2}^{*}}\left(\left\|\Phi_{1}\right\| \Psi_{1}(\|\varpi\|)\right)
\end{aligned}
$$

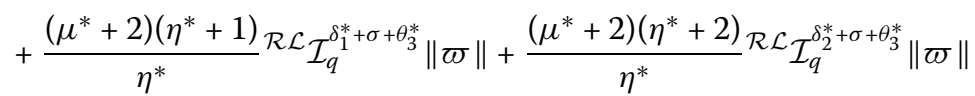

$$
\begin{aligned}
& \left.+\frac{\lambda_{1}^{*}\left(\mu^{*}+2\right)}{\eta^{*}} \mathcal{R} \mathcal{L}_{\mathcal{I}_{q}^{\gamma_{1}^{*}+\sigma+\theta_{3}^{*}}}\left(\left\|\Phi_{1}\right\| \Psi_{1}(\|\varpi\|)\right)\right] \\
& +\frac{\lambda_{2}^{*}}{\eta^{*}} \mathcal{R} \mathcal{L}_{\mathcal{I}_{q}^{\gamma_{2}^{*}+\sigma}}\left(\left\|\Phi_{2}\right\| \Psi_{2}(\|\varpi\|)\right)+\frac{1}{\tilde{\Xi}_{*}}\left[\frac { \lambda _ { 2 } ^ { * } \mu ^ { * } } { \eta ^ { * } } \mathcal { R } \mathcal { L } _ { \mathcal { I } _ { q } ^ { \gamma _ { 2 } ^ { * } + \sigma + \theta _ { 1 } ^ { * } } } \left(\left\|\Phi_{2}\right\| \Psi_{2}(\|\varpi\|)\right.\right. \\
& +\frac{\lambda_{2}^{*}\left(\mu^{*}+1\right)}{\eta^{*}} \mathcal{R L}_{\mathcal{I}_{q}^{\gamma_{2}^{*}+\sigma+\theta_{2}^{*}}}\left(\left\|\Phi_{2}\right\| \Psi_{2}(\|\varpi\|)\right. \\
& +\frac{\lambda_{2}^{*}\left(\mu^{*}+2\right)}{\eta^{*}} \mathcal{R} \mathcal{L}_{\mathcal{I}_{q}^{\gamma}}^{\gamma_{2}^{*}+\sigma+\theta_{3}^{*}}\left(\left\|\Phi_{2}\right\| \Psi_{2}(\|\varpi\|)\right] \\
& =\tilde{\Omega}_{*}^{(1)}\|\varpi\|+\tilde{\Omega}_{*}^{(2)}\left\|\Phi_{1}\right\| \Psi_{1}(\|\varpi\|)+\tilde{\Omega}_{*}^{(3)}\left\|\Phi_{2}\right\| \Psi_{2}(\|\varpi\|) .
\end{aligned}
$$

Hence, the above inequality yields

$$
\|\mathbb{A} \varpi\| \leq \tilde{\Omega}_{*}^{(1)} \tilde{R}+\tilde{\Omega}_{*}^{(2)}\left\|\Phi_{1}\right\| \Psi_{1}(\tilde{R})+\tilde{\Omega}_{*}^{(3)}\left\|\Phi_{2}\right\| \Psi_{2}(\tilde{R}) .
$$

This indicates that the operator $\mathbb{A}$ is uniformly bounded. In the second stage, we proceed to verify that $\mathbb{A}$ maps bounded sets (i.e., balls) into equicontinuous subsets of $\mathfrak{W}$. To see 
this, take $z, x \in[0,1]$ with $z<x$ and $\varpi \in \mathbb{B}_{\tilde{R}}$. In this case, we get

$$
\begin{aligned}
& |\mathbb{A} \varpi(x)-\mathbb{A} \varpi(z)| \\
& \leq \frac{\left(\eta^{*}+1\right) \tilde{R}}{\eta^{*} \Gamma_{q}\left(\delta_{1}^{*}+\sigma\right)} \\
& \times\left(\int_{0}^{z}\left[(x-q r)^{\left(\delta_{1}^{*}+\sigma-1\right)}-(z-q r)^{\left(\delta_{1}^{*}+\sigma-1\right)}\right] \mathrm{d}_{q} r+\int_{z}^{x}(x-q r)^{\left(\delta_{1}^{*}+\sigma-1\right)} \mathrm{d}_{q} r\right) \\
& +\frac{\left(\eta^{*}+2\right) \tilde{R}}{\eta^{*} \Gamma_{q}\left(\delta_{2}^{*}+\sigma\right)} \\
& \times\left(\int_{0}^{z}\left[(x-q r)^{\left(\delta_{2}^{*}+\sigma-1\right)}-(z-q r)^{\left(\delta_{2}^{*}+\sigma-1\right)}\right] \mathrm{d}_{q} r+\int_{z}^{x}(x-q r)^{\left(\delta_{2}^{*}+\sigma-1\right)} \mathrm{d}_{q} r\right) \\
& +\frac{\lambda_{1}^{*}\left\|\Phi_{1}\right\| \Psi_{1}(\tilde{R})}{\eta^{*} \Gamma_{q}\left(\gamma_{1}^{*}+\sigma\right)} \\
& \times\left(\int_{0}^{z}\left[(x-q r)^{\left(\gamma_{1}^{*}+\sigma-1\right)}-(z-q r)^{\left(\gamma_{1}^{*}+\sigma-1\right)}\right] \mathrm{d}_{q} r+\int_{z}^{x}(x-q r)^{\left(\gamma_{1}^{*}+\sigma-1\right)} \mathrm{d}_{q} r\right)
\end{aligned}
$$

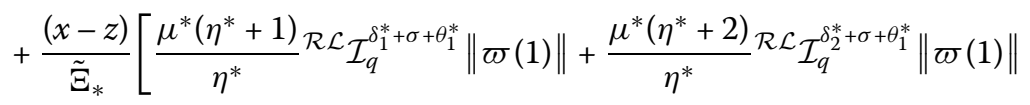

$$
\begin{aligned}
& +\frac{\lambda_{1}^{*} \mu^{*}}{\eta^{*}} \mathcal{R} \mathcal{L}_{\mathcal{I}_{q}^{\gamma}}^{\gamma_{1}^{*}+\sigma+\theta_{1}^{*}}\left\|\hat{h}_{*}(1)\right\|+\frac{\left(\mu^{*}+1\right)\left(\eta^{*}+1\right)}{\eta^{*}} \mathcal{R} \mathcal{L}_{\mathcal{I}_{q}^{\delta *}}^{\delta_{1}^{*}+\sigma+\theta_{2}^{*}}\|\varpi(1)\|
\end{aligned}
$$

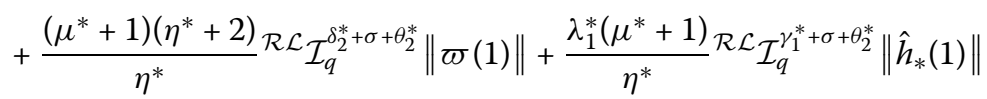

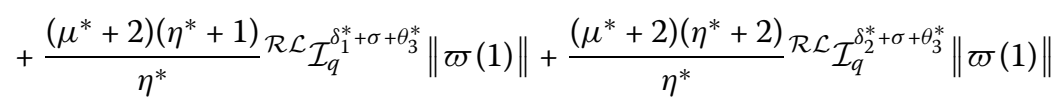

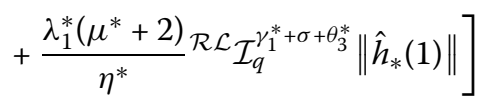

$$
\begin{aligned}
& +\frac{\lambda_{2}^{*}\left\|\Phi_{2}\right\| \Psi_{2}(\tilde{R})}{\eta^{*} \Gamma_{q}\left(\gamma_{2}^{*}+\sigma\right)} \\
& \times\left(\int_{0}^{z}\left[(x-q r)^{\left(\gamma_{2}^{*}+\sigma-1\right)}-(z-q r)^{\left(\gamma_{2}^{*}+\sigma-1\right)}\right] \mathrm{d}_{q} r+\int_{z}^{x}(x-q r)^{\left(\gamma_{2}^{*}+\sigma-1\right)} \mathrm{d}_{q} r\right) \\
& +\frac{(x-z)\left\|\Phi_{2}\right\| \Psi_{2}(\tilde{r})}{\tilde{\Xi}_{*}}\left[\frac{\lambda_{2}^{*} \mu^{*}}{\eta^{*} \Gamma_{q}\left(\gamma_{2}^{*}+\sigma+\theta_{1}^{*}\right)} \int_{0}^{1}(1-q r)^{\left(\gamma_{2}^{*}+\sigma+\theta_{1}^{*}-1\right)} \mathrm{d}_{q} r\right. \\
& +\frac{\lambda_{2}^{*}\left(\mu^{*}+1\right)}{\eta^{*} \Gamma_{q}\left(\gamma_{2}^{*}+\sigma+\theta_{2}^{*}\right)} \int_{0}^{1}(1-q r)^{\left(\gamma_{2}^{*}+\sigma+\theta_{2}^{*}-1\right)} \mathrm{d}_{q} r \\
& \left.+\frac{\lambda_{2}^{*}\left(\mu^{*}+2\right)}{\eta^{*} \Gamma_{q}\left(\gamma_{2}^{*}+\sigma+\theta_{3}^{*}\right)} \int_{0}^{1}(1-q r)^{\left(\gamma_{2}^{*}+\sigma+\theta_{3}^{*}-1\right)} \mathrm{d}_{q} r\right] \text {. }
\end{aligned}
$$

We find that the right-hand side of the obtained inequality is not dependent on $\varpi \in \mathbb{B}_{\tilde{R}}$ and also approaches 0 when $z$ tends to $x$. In consequence, $\mathbb{A}$ is equicontinuous, and hence we have confirmed the complete continuity of $\mathbb{A}: \mathfrak{W} \rightarrow \mathfrak{W}$ by the Arzelá-Ascoli theorem. Consequently, $\mathbb{A}$ is a compact operator.

Eventually, in order to finish checking all the assumptions of the Leray-Schauder nonlinear alternative (Theorem 2.3), it will be verified that the set of all obtained solutions of an operator equation $\varpi=\hat{c}(\mathbb{A} \varpi)$ is bounded for $\hat{c} \in[0,1]$. For this purpose, assume 
that $\varpi^{*}$ is a solution of equation $\varpi^{*}=\hat{c} \mathbb{A} \varpi^{*}$ for $\hat{c} \in[0,1]$. Then by utilizing the strategy applied in the first stage, for any $z \in[0,1]$, we have

$$
\left\|\varpi^{*}\right\| \leq \tilde{\Omega}_{*}^{(1)}\left\|\varpi^{*}\right\|+\tilde{\Omega}_{*}^{(2)}\left\|\Phi_{1}\right\| \Psi_{1}\left(\left\|\varpi^{*}\right\|\right)+\tilde{\Omega}_{*}^{(3)}\left\|\Phi_{2}\right\| \Psi_{2}\left(\left\|\varpi^{*}\right\|\right) .
$$

In this case, we get

$$
\frac{\left(1-\tilde{\Omega}_{*}^{(1)}\right)\left\|\varpi^{*}\right\|}{\tilde{\Omega}_{*}^{(2)}\left\|\Phi_{1}\right\| \Psi_{1}\left(\left\|\varpi^{*}\right\|\right)+\tilde{\Omega}_{*}^{(3)}\left\|\Phi_{2}\right\| \Psi_{2}\left(\left\|\varpi^{*}\right\|\right)} \leq 1
$$

In the light of hypothesis (HK4), we can find a real number $\mathfrak{N}>0$ so that $\left\|\varpi^{*}\right\| \neq \mathfrak{N}$. Now, we construct a set

$$
\mathbb{O}=\left\{\varpi^{*} \in \mathfrak{W}:\left\|\varpi^{*}\right\|<\mathfrak{N}\right\} .
$$

We simply see that $\mathbb{A}: \overline{\mathbb{O}} \rightarrow \mathfrak{W}$ is an operator which is continuous and completely continuous. In view of this choice of $\mathbb{O}$, we cannot find $\varpi^{*} \in \partial \mathbb{O}$ which satisfies an equation $\varpi^{*}=\hat{c}\left(\mathbb{A} \varpi^{*}\right)$ for some $\hat{c} \in(0,1)$. Finally, by the nonlinear alternative of Leray-Schauder type, we realize that the operator $\mathbb{A}$ has a fixed point belonging to $\overline{\mathbb{O}}$. In consequence, there is at least one solution on $[0,1]$ for the nonlinear Caputo fractional quantum multiintegro-differential problem (1).

In the following part of the present section, the uniqueness criterion for solutions of the given nonlinear Caputo fractional quantum multi-integro-differential problem (1) is checked with the aid of Banach contraction principle (Theorem 2.4).

Theorem 3.5 Suppose that $\hat{h}_{*}:[0,1] \times \mathfrak{W} \rightarrow \mathfrak{W}$ is a function which satisfies hypothesis (HK1). Moreover, let the following assumption be valid for the function $\hat{f}_{*}:[0,1] \times \mathfrak{W} \rightarrow \mathfrak{W}$ :

(HK5) there is a real constant $\mathfrak{K}>0$ so that for any $\varpi_{1}, \varpi_{2} \in \mathfrak{W}$, we have

$$
\left|\hat{f}_{*}\left(z, \varpi_{1}\right)-\hat{f}_{*}\left(z, \varpi_{2}\right)\right| \leq \mathfrak{K}\left|\varpi_{1}-\varpi_{2}\right|, \quad z \in[0,1] .
$$

Then there exists a unique solution on $[0,1]$ for the nonlinear Caputo fractional quantum multi-integro-differential problem (1) such that $\tilde{\Omega}_{*}^{(1)}+\hat{b}^{*} \tilde{\Omega}_{*}^{(2)}+\mathfrak{K} \tilde{\Omega}_{*}^{(3)}<1$, where $\tilde{\Omega}_{*}^{(1)}, \tilde{\Omega}_{*}^{(2)}$, and $\tilde{\Omega}_{*}^{(3)}$ are given by (12).

Proof By utilizing Theorem 2.4, we shall verify that $\mathbb{A}: \mathfrak{W} \rightarrow \mathfrak{W}$ defined by (11) is an operator having a unique fixed point which corresponds to a unique solution of the mentioned nonlinear Caputo fractional quantum multi-integro-differential problem (1). By taking $\sup _{z \in[0,1]}\left|\hat{h}_{*}(z, 0)\right|=\hat{H}_{*}<\infty, \sup _{z \in[0,1]}\left|\hat{f}_{*}(z, 0)\right|=\hat{F}_{*}<\infty$, choosing $\tilde{\varepsilon}>0$ so that

$$
\tilde{\varepsilon} \geq \frac{\hat{F}_{*} \tilde{\Omega}_{*}^{(3)}+\hat{H}_{*} \tilde{\Omega}_{*}^{(2)}}{1-\left(\tilde{\Omega}_{*}^{(1)}+\hat{b}^{*} \tilde{\Omega}_{*}^{(2)}+\mathfrak{K} \tilde{\Omega}_{*}^{(3)}\right)},
$$

and by constructing a bounded ball $\mathbb{B}_{\tilde{\varepsilon}}=\{\varpi \in \mathfrak{W}:\|\varpi\| \leq \tilde{\varepsilon}\}$, we claim that $\mathbb{A B}_{\tilde{\varepsilon}} \subset \mathbb{B}_{\tilde{\varepsilon}}$. For an arbitrary element $\varpi \in \mathbb{B}_{\tilde{\varepsilon}}$ and due to hypotheses (HK1) and (HK5), we get

$$
\|\mathbb{A} \varpi\| \leq \frac{\eta^{*}+1}{\eta^{*}} \mathcal{R} \mathcal{L}_{\mathcal{I}_{q}^{\delta^{*}+\sigma}\|\varpi\|}
$$




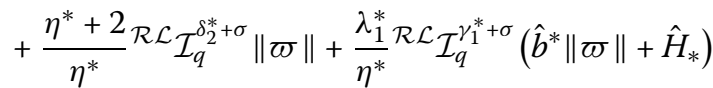

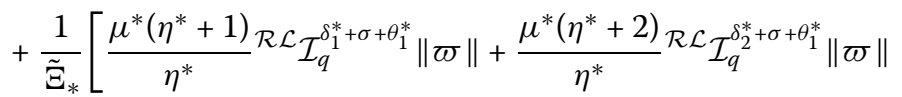

$$
\begin{aligned}
& +\frac{\lambda_{1}^{*} \mu^{*}}{\eta^{*}} \mathcal{R} \mathcal{L}_{\mathcal{I}_{q}^{\gamma_{1}^{*}+\sigma+\theta_{1}^{*}}}\left(\hat{b}^{*}\|\varpi\|+\hat{H}_{*}\right)+\frac{\left(\mu^{*}+1\right)\left(\eta^{*}+1\right)}{\eta^{*}} \mathcal{R} \mathcal{L}_{\mathcal{I}_{q}^{\delta_{1}^{*}+\sigma+\theta_{2}^{*}}\|\varpi\|}
\end{aligned}
$$

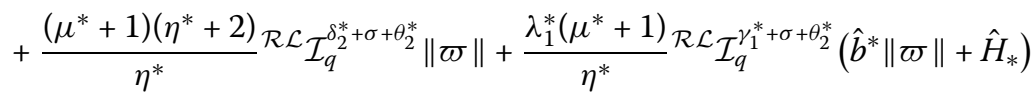

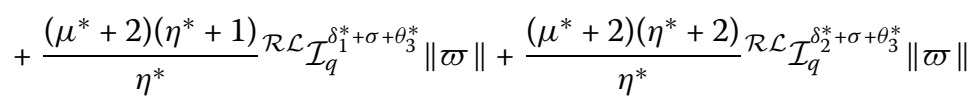

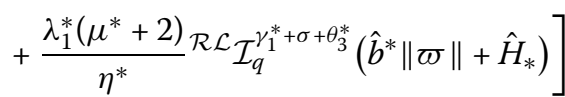

$$
\begin{aligned}
& +\frac{\lambda_{2}^{*}}{\eta^{*}} \mathcal{R} \mathcal{L}_{\mathcal{I}_{q}^{\gamma}}^{\gamma_{2}^{*}+\sigma}\left(\mathfrak{K}\|\varpi\|+\hat{F}_{*}\right)+\frac{1}{\tilde{\Xi}_{*}}\left[\frac{\lambda_{2}^{*} \mu^{*}}{\eta^{*}} \mathcal{R} \mathcal{L}_{\mathcal{I}_{q}^{\gamma_{2}^{*}+\sigma+\theta_{1}^{*}}}\left(\mathfrak{K}\|\varpi\|+\hat{F}_{*}\right)\right. \\
& +\frac{\lambda_{2}^{*}\left(\mu^{*}+1\right)}{\eta^{*}} \mathcal{R} \mathcal{L}_{\mathcal{I}_{q}^{\gamma}}^{\gamma_{2}^{*}+\sigma+\theta_{2}^{*}}\left(\mathfrak{K}\|\varpi\|+\hat{F}_{*}\right) \\
& \left.+\frac{\lambda_{2}^{*}\left(\mu^{*}+2\right)}{\eta^{*}} \mathcal{R} \mathcal{L}_{\mathcal{I}^{\prime}}^{\gamma_{2}^{*}+\sigma+\theta_{3}^{*}}\left(\mathfrak{K}\|\varpi\|+\hat{F}_{*}\right)\right] \\
& \leq\left(\tilde{\Omega}_{*}^{(1)}+\hat{b}^{*} \tilde{\Omega}_{*}^{(2)}+\mathfrak{K} \tilde{\Omega}_{*}^{(3)}\right) \tilde{\varepsilon}+\tilde{\Omega}_{*}^{(3)} \hat{F}_{*}+\tilde{\Omega}_{*}^{(2)} \hat{H}_{*}<\tilde{\varepsilon} .
\end{aligned}
$$

In view of the above result, it is seen that the claim is valid, and so we have $\mathbb{A B}_{\tilde{\varepsilon}} \subset \mathbb{B}_{\tilde{\varepsilon}}$. To confirm that the operator $\mathbb{A}: \mathfrak{W} \rightarrow \mathfrak{W}$ given by (11) is a contraction, let us assume that $z \in[0,1]$ and $\varpi_{1}, \varpi_{2} \in \mathfrak{W}$ are arbitrary. Now, by some straightforward computations, we can simply observe that

$$
\left\|\left(\mathbb{A} \varpi_{1}\right)-\left(\mathbb{A} \varpi_{2}\right)\right\| \leq\left(\tilde{\Omega}_{*}^{(1)}+\hat{b}^{*} \tilde{\Omega}_{*}^{(2)}+\mathfrak{K} \tilde{\Omega}_{*}^{(3)}\right)\left\|\varpi_{1}-\varpi_{2}\right\| .
$$

By invoking the hypothesis $\tilde{\Omega}_{*}^{(1)}+\hat{b}^{*} \tilde{\Omega}_{*}^{(2)}+\mathfrak{K} \tilde{\Omega}_{*}^{(3)}<1$, we conclude that $\mathbb{A}$ is a contraction. Therefore, as a conclusion of Theorem 2.4, $\mathbb{A}$ has a unique fixed point. In consequence, there exists a unique solution for the nonlinear Caputo fractional quantum multi-integrodifferential problem (1), and this ends the argument.

\section{Examples}

In the current section of this manuscript, three illustrative numerical examples are considered to examine our theoretical and analytical findings by using the proposed methods.

Example 4.1 (Illustration of Theorem 3.3) With due attention to the defined structure for the proposed quantum multi-integro-differential problem (1), we here design the following multi-order Caputo fractional quantum multi-integro-differential boundary value problem:

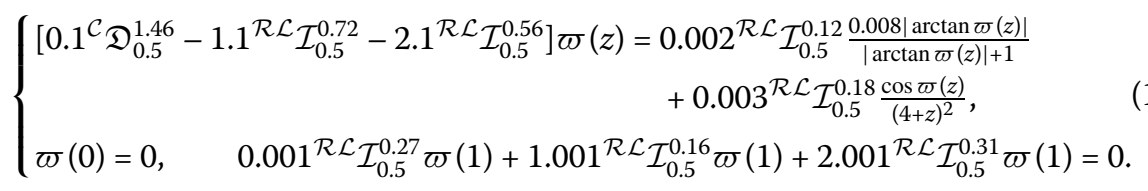


Here, we consider some constants as follows: $z=[0,1], \eta^{*}=0.1, \mu^{*}=0.001, \lambda_{1}^{*}=0.002$, $\lambda_{2}^{*}=0.003, \sigma=1.46, q=0.5, \delta_{1}^{*}=0.72, \delta_{2}^{*}=0.56, \gamma_{1}^{*}=0.12, \gamma_{2}^{*}=0.18, \theta_{1}^{*}=0.27, \theta_{2}^{*}=0.16$, and $\theta_{3}^{*}=0.31$. Further, two continuous functions $\hat{h}_{*}, \hat{f}_{*}:[0,1] \times \mathbb{R} \rightarrow \mathbb{R}$ are formulated by follows:

$$
\hat{h}_{*}(z, \varpi(z))=\frac{0.008|\arctan \varpi(z)|}{|\arctan \varpi(z)|+1}, \quad \hat{f}_{*}(z, \varpi(z))=\frac{\cos \varpi(z)}{(4+z)^{2}} .
$$

Notice that for each $\varpi_{1}, \varpi_{2} \in \mathbb{R}$, we get

$$
\begin{aligned}
\left|\hat{h}_{*}\left(z, \varpi_{1}\right)-\hat{h}_{*}\left(z, \varpi_{2}\right)\right| & \leq\left|\frac{0.008\left|\arctan \varpi_{1}(z)\right|}{\left|\arctan \varpi_{1}(z)\right|+1}-\frac{0.008\left|\arctan \varpi_{2}(z)\right|}{\left|\arctan \varpi_{2}(z)\right|+1}\right| \\
& \leq \frac{8}{1000}\left|\arctan \varpi_{1}(z)-\arctan \varpi_{2}(z)\right| \\
& \leq \frac{8}{1000}\left|\varpi_{1}(z)-\varpi_{2}(z)\right| .
\end{aligned}
$$

Thus we get $\left|\hat{h}_{*}\left(z, \varpi_{1}\right)-\hat{h}_{*}\left(z, \varpi_{2}\right)\right| \leq 0.008\left|\varpi_{1}(z)-\varpi_{2}(z)\right|$ so that $\hat{b}^{*}=0.008>0$. Furthermore, there is a continuous function $\Upsilon(z)=\frac{1}{(4+z)^{2}}$ on the interval $[0,1]$ so that an inequality $\left|\hat{f}_{*}(z, \varpi(z))\right| \leq\left|\frac{\cos \varpi(z)}{(4+z)^{2}}\right| \leq \Upsilon(z)$ holds for any $\varpi \in \mathbb{R}$. In this case, we have $\|\Upsilon\|=\sup _{z \in[0,1]} \Upsilon(z)=0.0625$. By utilizing the above-given values, it is immediately obtained that $\tilde{\Xi}_{*}=0.0538, \tilde{\Omega}_{*}^{(1)}=0.095734, \tilde{\Omega}_{*}^{(2)}=0.000000292$, and $\tilde{\Omega}_{*}^{(3)}=0.00000042$. Hence we reach required value $\tilde{\Omega}_{*}^{(1)}+\hat{b}^{*} \tilde{\Omega}_{*}^{(2)}=0.0957340023<1$. It is observed that all the hypotheses of Theorem 3.3 are valid for this problem. In consequence, the conclusion of Theorem 3.3 yields that the nonlinear multi-order Caputo fractional quantum multiintegro-differential boundary problem (13) has at least one solution on $[0,1]$.

Example 4.2 (Illustration of Theorem 3.4) With due attention to the defined structure for the proposed quantum multi-integro-differential problem (1), we here consider the following nonlinear multi-order Caputo fractional quantum multi-integro-differential boundary value problem:

$$
\left\{\begin{array}{l}
{\left[0.1^{\mathcal{C}} \mathfrak{D}_{0.5}^{1.46}-1.1^{\mathcal{R} \mathcal{L}} \mathcal{I}_{0.5}^{0.72}-2.1^{\mathcal{R} \mathcal{L}} \mathcal{I}_{0.5}^{0.56}\right] \varpi(z)} \\
=0.002^{\mathcal{R} \mathcal{L}} \mathcal{I}_{0.5}^{0.12} \frac{1}{\sqrt{64+z^{3}}} \\
\quad \times\left(\cos \varpi(z)+\frac{|\sin \varpi(z)|}{1+|\sin \varpi(z)|}\right)+0.003^{\mathcal{R} \mathcal{L}} \mathcal{I}_{0.5}^{0.18} \frac{1}{z+5}\left(\frac{1}{4}+\frac{|\arcsin \varpi(z)|}{1+|\arcsin \varpi(z)|}\right) \\
\varpi(0)=0, \quad 0.001^{\mathcal{R} \mathcal{L}} \mathcal{I}_{0.5}^{0.27} \varpi(1)+1.001^{\mathcal{R} \mathcal{L}} \mathcal{I}_{0.5}^{0.16} \varpi(1)+2.001^{\mathcal{R}} \mathcal{L} \mathcal{I}_{0.5}^{0.31} \varpi(1)=0
\end{array}\right.
$$

such that $z=[0,1], \eta^{*}=0.1, \mu^{*}=0.001, \lambda_{1}^{*}=0.002, \lambda_{2}^{*}=0.003, \sigma=1.46, q=0.5, \delta_{1}^{*}=0.72$, $\delta_{2}^{*}=0.56, \gamma_{1}^{*}=0.12, \gamma_{2}^{*}=0.18, \theta_{1}^{*}=0.27, \theta_{2}^{*}=0.16$, and $\theta_{3}^{*}=0.31$. Moreover, the functions $\hat{h}_{*}, \hat{f}_{*}:[0,1] \times \mathbb{R} \rightarrow \mathbb{R}$ defined by

$$
\hat{h}_{*}(z, \varpi(z))=\frac{1}{\sqrt{64+z^{3}}}\left(\cos \varpi(z)+\frac{|\sin \varpi(z)|}{1+|\sin \varpi(z)|}\right)
$$

and

$$
\hat{f}_{*}(z, \varpi(z))=\frac{1}{z+5}\left(\frac{1}{4}+\frac{|\arcsin \varpi(z)|}{1+|\arcsin \varpi(z)|}\right)
$$


are continuous. Evidently, we have the following inequalities:

$$
\left|\hat{h}_{*}(z, \varpi(z))\right| \leq \frac{1}{\sqrt{64+z^{3}}}(1+\|\varpi\|), \quad\left|\hat{f}_{*}(z, \varpi(z))\right| \leq \frac{1}{z+5}(1+\|\varpi\|) .
$$

Thus, we take $\Phi_{1}(z)=\frac{1}{\sqrt{64+z^{3}}}$ and $\Phi_{2}(z)=\frac{1}{z+5}$ and $\Psi_{1}(\|\varpi\|)=\Psi_{2}(\|\varpi\|)=1+\|\varpi\|$. Notice that $\left\|\Phi_{1}\right\|=\frac{1}{8}=0.125,\left\|\Phi_{2}\right\|=\frac{1}{5}=0.2$, and $\Psi_{1}(\mathfrak{N})=\Psi_{2}(\mathfrak{N})=1+\mathfrak{N}$. In view of the above data, we find that $\tilde{\Xi}_{*}=0.0538, \tilde{\Omega}_{*}^{(1)}=0.095734<1, \tilde{\Omega}_{*}^{(2)}=0.000000292$, and $\tilde{\Omega}_{*}^{(3)}=0.00000042$. Therefore, by taking into account hypothesis (HK4), we find that $\mathfrak{N}>0.00000013325=1.3325 \times 10^{-7}$. At this point, we see that all the hypotheses of Theorem 3.4 hold for this problem. Therefore, by Theorem 3.4, the nonlinear multi-order Caputo fractional quantum multi-integro-differential boundary problem (14) has at least one solution on $[0,1]$.

Example 4.3 (Illustration of Theorem 3.5) With due attention to the defined structure for the proposed quantum multi-integro-differential problem (1), we here design the following multi-order Caputo fractional quantum multi-integro-differential boundary value problem:

$$
\left\{\begin{array}{l}
{\left[0.1^{\mathcal{C}} \mathfrak{D}_{0.5}^{1.46}-1.1^{\mathcal{R} \mathcal{L}} \mathcal{I}_{0.5}^{0.72}-2.1^{\mathcal{R} \mathcal{L}} \mathcal{I}_{0.5}^{0.56}\right] \varpi(z)} \\
\quad=0.002^{\mathcal{R}} \mathcal{L} \mathcal{I}_{0.5}^{0.12} \frac{0.005 \mid \cos (\pi z))|| \varpi(z) \mid}{|\varpi(z)|+1}+0.003^{\mathcal{R}} \mathcal{L} \mathcal{I}_{0.5}^{0.18} \frac{2|\varpi(z)|}{125+125|\varpi(z)|)} \\
\varpi(0)=0, \quad 0.001^{\mathcal{R} \mathcal{L}} \mathcal{I}_{0.5}^{0.27} \varpi(1)+1.001^{\mathcal{R} \mathcal{L}} \mathcal{I}_{0.5}^{0.16} \varpi(1)+2.001^{\mathcal{R}} \mathcal{L} \mathcal{I}_{0.5}^{0.31} \varpi(1)=0
\end{array}\right.
$$

such that $z=[0,1], \eta^{*}=0.1, \mu^{*}=0.001, \lambda_{1}^{*}=0.002, \lambda_{2}^{*}=0.003, \sigma=1.46, q=0.5, \delta_{1}^{*}=0.72$, $\delta_{2}^{*}=0.56, \gamma_{1}^{*}=0.12, \gamma_{2}^{*}=0.18, \theta_{1}^{*}=0.27, \theta_{2}^{*}=0.16$, and $\theta_{3}^{*}=0.31$. Besides, two functions $\hat{h}_{*}, \hat{f}_{*}:[0,1] \times \mathbb{R} \rightarrow \mathbb{R}$ defined by

$$
\hat{h}_{*}(z, \varpi(z))=\frac{0.005 \mid \cos (\pi z))|| \varpi(z) \mid}{|\varpi(z)|+1}, \quad \hat{f}_{*}(z, \varpi(z))=\frac{2|\varpi(z)|}{125+125|\varpi(z)|)}
$$

are supposed to be continuous on the relevant domain. Then we get $\hat{b}^{*}=0.005$ and $\mathfrak{K}=$ 0.016 , since one can simply see that

$$
\left|\hat{h}_{*}\left(z, \varpi_{1}(z)\right)-\hat{h}_{*}\left(z, \varpi_{2}(z)\right)\right| \leq 0.005\left(\left|\varpi_{1}(z)-\varpi_{2}(z)\right|\right)
$$

and

$$
\left|\hat{f}_{*}\left(z, \varpi_{1}(z)\right)-\hat{f}_{*}\left(z, \varpi_{2}(z)\right)\right| \leq 0.016\left(\left|\varpi_{1}(z)-\varpi_{2}(z)\right|\right) .
$$

Eventually, in the light of the above assumptions, we find that $\tilde{\Xi}_{*}=0.0538$ and

$$
\tilde{\Omega}_{*}^{(1)}+\hat{b}^{*} \tilde{\Omega}_{*}^{(2)}+\mathfrak{K} \tilde{\Omega}_{*}^{(3)}=0.095734009<1 .
$$

In consequence, we conclude that all the hypotheses of Theorem 3.5 hold for this problem. Therefore, by Theorem 3.5, the nonlinear multi-order Caputo fractional quantum multiintegro-differential boundary problem (15) has at least one solution on $[0,1]$. 
Table 1 Numerical values of $\tilde{\Omega}_{*}^{(1)}, \tilde{\Omega}_{*}^{(2)}$, and $\tilde{\Omega}_{*}^{(3)}$ for $q=0.3,0.5,0.7$

\begin{tabular}{llll}
\hline & $q=0.3$ & $q=0.5$ & $q=0.7$ \\
\hline$\tilde{\Omega}_{*}^{(1)}$ & 0.075999 & 0.095734 & 0.012214 \\
$\tilde{\Omega}_{*}^{(2)}$ & 0.0000011 & 0.000000292 & 0.00000016 \\
$\tilde{\Omega}_{*}^{(3)}$ & 0.0000017 & 0.00000042 & 0.00000023 \\
\hline
\end{tabular}

Remark 4.4 Notice that one can find other values of three nonzero constants $\tilde{\Omega}_{*}^{(1)}, \tilde{\Omega}_{*}^{(2)}$, and $\tilde{\Omega}_{*}^{(3)}$ for different values of $q=0.3,0.5,0.7$ in Table 1 . Indeed, we only calculated required numerical data of above examples for $q=0.5$.

\section{Conclusion}

As years and even decades go by, the human beings need to be acquainted with different natural phenomena more and more. One possible way to achieve this purpose is to study the mathematical structures of these processes by means of the logical techniques and tools available in mathematics. In the present framework of this research manuscript, we formulate a new generalized structure of the nonlinear Caputo fractional quantum multiintegro-differential equation in which such multi-order structure of quantum integrals are considered for the first time. In fact, in the light of this type of boundary value problem equipped with the multi-integro-differential setting, one can simply study different cases of the existing usual integro-differential problems in the literature. In this direction, we utilize well-known analytical techniques to derive desired criteria which guarantee the existence of solutions for the proposed multi-order quantum multi-integro-differential problem. Further, some numerical examples are provided to examine our theoretical and analytical findings based on the proposed methods.

Acknowledgements

The second author was supported by Dicle University. The third and fourth authors were supported by Azarbaijan Shahid Madani University. This research is supported by Industrial University of Ho Chi Minh City (IUH) under grant number 66/HD-DHCN. The authors express their gratitude to dear unknown referees for their helpful suggestions which improved the final version of this paper.

Funding

Not applicable.

Availability of data and materials

Data sharing not applicable to this article as no datasets were generated or analyzed during the current study.

Ethics approval and consent to participate

Not applicable.

Competing interests

The authors declare that they have no competing interests.

Consent for publication

Not applicable.

Authors' contributions

The authors declare that the study was realized in collaboration with equal responsibility. All authors read and approved the final manuscript.

Authors' information

(Nguyen Duc Phuong: nguyenducphuong@iuh.edu.vn and Fethiye Muge Sakar: mugesakar@hotmail.com and Sine Etemad: sina.etemad@gmail.com) 


\section{Author details}

${ }^{1}$ Faculty of Fundamental Science, Industrial University of Ho Chi Minh City, Ho Chi Minh City 700000, Vietnam.

${ }^{2}$ Department of Business Administration, Faculty of Management and Economics, Dicle University, Diyarbakir, Turkey.

${ }^{3}$ Department of Mathematics, Azarbaijan Shahid Madani University, Tabriz, Iran. ${ }^{4}$ Institute of Research and Development, Duy Tan University, Da Nang 550000, Vietnam. ${ }^{5}$ Faculty of Natural Sciences, Duy Tan University, Da Nang 550000, Vietnam. ${ }^{6}$ Department of Medical Research, China Medical University Hospital, China Medical University, Taichung, Taiwan.

\section{Publisher's Note}

Springer Nature remains neutral with regard to jurisdictional claims in published maps and institutional affiliations.

Received: 20 August 2020 Accepted: 1 November 2020 Published online: 11 November 2020

\section{References}

1. Baleanu, D., Etemad, S., Rezapour, S.: A hybrid Caputo fractional modeling for thermostat with hybrid boundary value conditions. Bound. Value Probl. 2020, 64 (2020). https://doi.org/10.1186/s13661-020-01361-0

2. Baleanu, D., Jajarmi, A., Mohammadi, H., Rezapour, S.: Analysis of the human liver model with Caputo-Fabrizio fractional derivative. Chaos Solitons Fractals 134, 109705 (2020)

3. Khan, S.A., Shah, K., Zaman, G., Jarad, F.: Existence theory and numerical solutions to smoking model under Caputo-Fabrizio fractional derivative. Chaos 29, 013128 (2019). https://doi.org/10.1063/1.5079644

4. Baleanu, D., Aydogan, S.M., Mohammadi, H., Rezapour, S.: On modelling of epidemic childhood diseases with the Caputo-Fabrizio derivative by using the Laplace Adomian decomposition method. Alex. Eng. J. (2020). https://doi.org/10.1016/j.aej.2020.05.007

5. Baleanu, D., Rezapour, S., Saberpour, Z:: On fractional integro-differential inclusions via the extended fractional Caputo-Fabrizio derivation. Bound. Value Probl. 2019, 79 (2019)

6. Tuan, N.H., Mohammadi, H., Rezapour, S.: A mathematical model for COVID-19 transmission by using the Caputo fractional derivative. Chaos Solitons Fractals 140, 110107 (2020). https://doi.org/10.1016/j.chaos.2020.110107

7. Alsaedi, A., Baleanu, D., Etemad, S., Rezapour, S.: On coupled systems of time-fractional differential problems by using a new fractional derivative. J. Funct. Spaces 2016, Article ID 4626940 (2016). https://doi.org/10.1155/2016/4626940

8. Baleanu, D., Etemad, S., Rezapour, S.: On a fractional hybrid integro-differential equation with mixed hybrid integral boundary value conditions by using three operators. Alex. Eng. J. 59(5), 3019-3027 (2020) https://doi.org/10.1016/j.aej.2020.04.053

9. Baleanu, D., Rezapour, S., Mohammadi, H.: Some existence results on nonlinear fractional differential equations. Philos. Trans. - Royal Soc., Math. Phys. Eng. Sci. 371, 20120144 (2013). https://doi.org/10.1098/rsta.2012.0144

10. Baleanu, D., Mousalou, A., Rezapour, S.: On the existence of solutions for some infinite coefficient-symmetric Caputo-Fabrizio fractional integro-differential equations. Bound. Value Probl. 2017, 145 (2017). https://doi.org/10.1186/s13661-017-0867-9

11. Baleanu, D., Etemad, S., Rezapour, S., Alsaedi, A.: On a time-fractional integro-differential equation via three-point boundary value conditions. J. Funct. Spaces 2015, Article ID 785738 (2015). https://doi.org/10.1155/2015/785738

12. Benchohra, M., Lazreg, J.E.: Existence and Ulam stability for non-linear implicit fractional differential equations with Hadamard derivative. Stud. Univ. Babeş-Bolyai, Math. 62(1), 27-38 (2017)

13. Etemad, S., Ntouyas, S.K., Tariboon, J.: Existence results for three-point boundary value problems for nonlinear fractional differential equations. J. Nonlinear Sci. Appl. 9(8), 2105-2116 (2016). https://doi.org/10.22436/jnsa.009.05.16

14. $\mathrm{Ma}, \mathrm{X} .$, Huang, C.: Numerical solution of fractional integro-differential equations by a hybrid collocation method. Appl. Math. Comput. 219(12), 6750-6760 (2013). https://doi.org/10.1016/j.amc.2012.12.072

15. Ntouyas, S.K., Etemad, S.: On the existence of solutions for fractional differential inclusions with sum and integral boundary conditions. Appl. Math. Comput. 266, 235-243 (2015). https://doi.org/10.1016/j.amc.2015.05.036

16. Ntouyas, S.K., Etemad, S., Tariboon, J.: Existence of solutions for fractional differential inclusions with integra boundary conditions. Bound. Value Probl. 2015, 92 (2015). https://doi.org/10.1186/s13661-015-0356-y

17. Thabet, S.T.M., Dhakne, M.B.: On abstract fractional integro-differential equations via measure of noncompactness. Adv. Fixed Point Theory 6(2), 175-193 (2016)

18. Wang, G., Pei, K., Agarwal, R.P., Zhang, L., Ahmad, B.: On the existence of solutions for fractional differential inclusions with sum and integral boundary conditions. J. Comput. Appl. Math. 343, 230-239 (2018). https://doi.org/10.1016/j.cam.2018.04.062

19. Jackson, F.H.: On q-definite integrals. Q. J. Pure Appl. Math. 41, 193-203 (1910)

20. Adams, C.R.: Linear q-difference equations. Bull. Am. Math. Soc. 37(6), 361-400 (1931)

21. Adams, C.R.: Note on the integro-q-difference equations. Trans. Am. Math. Soc. 31(4), 861-867 (1929). https://doi.org/10.2307/1989569

22. Adams, C.R.: The general theory of a class of linear partial q-difference equations. Trans. Am. Math. Soc. 26(3), 283-312 (1924). https://doi.org/10.2307/1989141

23. Carman, M.G.: Expansion problems in connection with homogeneous linear $q$-difference equations. Trans. Am. Math. Soc. 28(3), 523-535 (1926). https://doi.org/10.2307/1989194

24. Starcher, G.W.: On identities arising from solutions of $q$-difference equations and some interpretations in number theory. Am. J. Math. 53(4), 801-816 (1931). https://doi.org/10.2307/2371227

25. Trjitzinsky, W.J.: Analytic theory of linear q-difference equations. Acta Math. 61, 1-38 (1933). https://doi.org/10.1007/BF02547785

26. Trjitzinsky, W.J.: Theory of nonlinear q-difference systems. Ann. Mat. Pura Appl. 17, 59-106 (1938). https://doi.org/10.1007/BF02410696

27. Abdi, W.H.: On certain q-difference equations and q-Laplace transform. Proc. Natl. Acad. Sci. India Sect. A Phys. Sci. 28 $1-15(1962)$

28. Miller, W.J.: Lie theory and q-difference equations. SIAM J. Math. Anal. 1(2), 171-188 (1970) https://doi.org/10.1137/0501017 
29. Abdeljawad, T., Alzabut, J.: The $q$-fractional analogue for Gronwall-type inequality. J. Funct. Spaces 2013, Article ID 543839 (2013). https://doi.org/10.1155/2013/543839

30. Ahmad, B., Etemad, S., Ettefagh, M., Rezapour, S.: On the existence of solutions for fractional $q$-difference inclusions with q-anti-periodic boundary conditions. Bull. Math. Soc. Sci. Math. Roum. 59(107)(2), 119-134 (2016)

31. Ahmad, B., Nieto, J.J., Alsaedi, A., Al-Hutami, H.: Boundary value problems of nonlinear fractional $q$-difference (integral) equations with two fractional orders and four-point nonlocal integral boundary conditions. Math. Proc. Camb. Philos. Soc. 28(8), 1719-1736 (2014). https://doi.org/10.2298/FIL1408719A

32. Aydogan, S.M., Baleanu, D., Aguilar, J.F.G., Rezapour, S., Samei, M.E.: Approximate endpoint solutions for a class of fractional $q$-differential inclusions. Fractals 28(8), 2040029 (2020). https://doi.org/10.1142/S0218348X20400290

33. Butt, R.I., Abdeljawad, T., Alqudah, M.A., Rehman, M.U.: Ulam stability of Caputo 9 -fractional delay difference equation: q-fractional Gronwall inequality approach. J. Inequal. Appl. 2019, 305 (2019). https://doi.org/10.1186/s13660-019-2257-6

34. Dumrongpokaphan, T., Patanarapeelert, N., Sitthiwirattham, T:: On sequential fractional $q$-Hahn integro-difference equations. Mathematics 8(5), 753 (2020). https://doi.org/10.3390/math8050753

35. Etemad, S., Ettefagh, M., Rezapour, S.: On the existence of solutions for nonlinear fractional $q$-difference equations with q-integral boundary conditions. J. Adv. Math. Stud. 8(2), 265-285 (2015)

36. Etemad, S., Ntouyas, S.K.: Application of the fixed point theorems on the existence of solutions for $q$-fractional boundary value problems. AIMS Math. 4(3), 997-1018 (2019). https://doi.org/10.3934/math.2019.3.997

37. Liang, S., Zhang, J.: Existence and uniqueness of positive solutions for three-point boundary value problem with fractional q-differences. J. Appl. Math. Comput. 40, 277-288 (2012). https://doi.org/10.1007/s12190-012-0551-2

38. Ouncharoen, R., Patanarapeelert, N., Sitthiwirattham, T.: Nonlocal $q$-symmetric integral boundary value problem for sequential q-symmetric integro-difference equations. Mathematics 6(11), 218 (2018). https://doi.org/10.3390/math6110218

39. Patanarapeelert, N., Sitthiwirattham, T.: On four-point fractional $q$-integro-difference boundary value problems involving separate nonlinearity and arbitrary fraction order. Bound. Value Probl. 2018, 41 (2018). https://doi.org/10.1186/s13661-018-0962-6

40. Sitthiwirattham, T:: On nonlocal fractional $q$-integral boundary value problems of fractional $q$-difference and fractional $q$-integro-difference equations involving different numbers of order and $q$. Bound. Value Probl. 2016, 12 (2016). https://doi.org/10.1186/s13661-016-0522-x

41. Baleanu, D., Nazemi, Z., Rezapour, S.: Attractivity for a k-dimensional system of fractional functional differential equations and global attractivity for a $k$-dimensional system of nonlinear fractional differential equations. J. Inequal. Appl. 2014, 31 (2014). https://doi.org/10.1186/1029-242X-2014-31

42. Ghorbanian, R., Hedayati, V., Postolache, M., Rezapour, S.: Attractivity for a k-dimensional system of fractional functional differential equations and global attractivity for a $k$-dimensional system of nonlinear fractional differential equations. J. Inequal. Appl. 2014, 319 (2014). https://doi.org/10.1186/1029-242X-2014-319

43. Agarwal, R.P., Baleanu, D., Hedayati, V., Rezapour, S.: Two fractional derivative inclusion problems via integral boundary conditions. Appl. Math. Comput. 257, 205-212 (2015). https://doi.org/10.1016/j.amc.2014.10.082

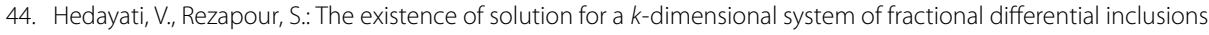
with anti-periodic boundary value problems. Filomat 30(6), 1601-1613 (2016). https://doi.org/10.2298/FIL1606601H

45. Aydogan, S.M., Nazemi, Z., Rezapour, S.: Positive solutions for a sum-type singular fractional integro-differential equation with m-point boundary conditions. Sci. Bull. "Politeh." Univ. Buchar., Ser. A, Appl. Math. Phys. 79(1), 89-98 (2017)

46. Etemad, S., Rezapour, S., Samei, M.E.: On a fractional Caputo-Hadamard inclusion problem with sum boundary value conditions by using approximate endpoint property. Math. Methods Appl. Sci. (2020). https://doi.org/10.1002/mma.6644

47. Rezapour, S., Samei, M.E.: On the existence of solutions for a multi-singular pointwise defined fractional q-integro-differential equation. Bound. Value Probl. 2020, 38 (2020). https://doi.org/10.1186/s13661-020-01342-3

48. Samei, M.E., Rezapour, S.: On a system of fractional $q$-differential inclusions via sum of two multi-term functions on a time scale. Bound. Value Probl. 2020, 135 (2020). https://doi.org/10.1186/s13661-020-01433-1

49. Zhou, W.X., Liu, H.Z.: Existence solutions for boundary value problem of nonlinear fractional $q$-difference equations. Adv. Differ. Equ. 2013, 113 (2013). https://doi.org/10.1186/1687-1847-2013-113

50. Niyom, S., Ntouyas, S.K., Laoprasittichok, S., Tariboon, J.: Boundary value problems with four orders of Riemann-Liouville fractional derivatives. Adv. Differ. Equ. 2016, 165 (2016). https://doi.org/10.1186/s13662-016-0897-0

51. Etemad, S., Ntouyas, S.K., Ahmad, B.: Existence theory for a fractional $q$-integro-difference equation with $q$-integral boundary conditions of different orders. Mathematics 7(8), 659 (2019). https://doi.org/10.3390/math7080659

52. Rajkovic, P.M., Marinkovic, S.D., Stankovic, M.S.: Fractional integrals and derivatives in q-calculus. Appl. Anal. Discrete Math. 1(1), 311-323 (2007) www.jstor.org/stable/43666058

53. Ferreira, R.A.C.: Positive solutions for a class of boundary value problems with fractional $q$-differences. Comput. Math. Appl. 61(2), 367-373 (2011). https://doi.org/10.1016/j.camwa.2010.11.012

54. Graef, J.R., Kong, L.: Positive solutions for a class of higher order boundary value problems with fractional q-derivatives. Appl. Math. Comput. 218(19), 9682-9689 (2012). https://doi.org/10.1016/j.amc.2012.03.006

55. Agarwal, R.P.: Certain fractional q-integrals and q-derivatives. Math. Proc. Camb. Philos. Soc. 66(2), 365-370 (1969). https://doi.org/10.1017/S0305004100045060

56. El-Shahed, M., Al-Askar, F.: Positive solutions for boundary value problem of nonlinear fractional $q$-difference equation. Int. Sch. Res. Netw. 2011, Article ID 385459 (2011). https://doi.org/10.5402/2011/385459

57. Krasnoselskii, M.A.: Two remarks on the method of successive approximations. Usp. Mat. Nauk 1, 123-127 (1955)

58. Granas, A., Dugundji, J.: Fixed Point Theory. Springer, New York (2003)

59. Deimling, K.: Nonlinear Functional Analysis. Springer, New York (1985) 